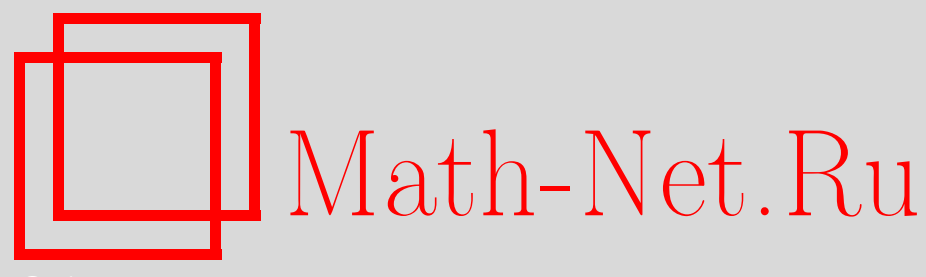

М. Бертола, Д. А. Короткин, Дискриминантные расслоения окружностей над локальными моделями графов Штребеля и кривых Бутру, ТМФ, 2018, том 197, номер 2, 163-207

DOI: https://doi.org/10.4213/tmf9513

Использование Общероссийского математического портала Math-Net.Ru подразумевает, что вы прочитали и согласны с пользовательским соглашением http://www . mathnet.ru/rus/agreement

Параметры загрузки:

IP : 54.210 .77 .194

26 апреля 2023 г., 18:08:18

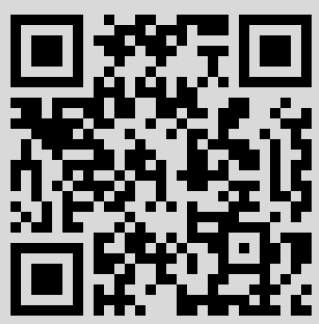




\title{
ФИЗИКА
}

Том 197, № 2

ноябрь, 2018

(C) 2018 г. М. Бертола*†, Д. А. Короткин*

\section{ДИСКРИМИНАНТНЫЕ РАССЛОЕНИЯ ОКРУЖНОСТЕЙ НАД ЛОКАЛЬНЫМИ МОДЕЛЯМИ ГРАФОВ ШТРЕБЕЛЯ И КРИВЫХ БУТРУ}

\begin{abstract}
Изучаются специальные "дискриминантные" расслоения окружностей над двумя элементарными пространствами $\mathcal{Q}_{0}^{\mathbb{R}}(-7)$ и $\mathcal{Q}_{0}^{\mathbb{R}}\left([-3]^{2}\right)$ модулей мероморфных квадратичных дифференциалов с вещественными периодами. Пространство $\mathcal{Q}_{0}^{\mathbb{R}}(-7)$ - это пространство модулей мероморфных квадратичных дифференциалов на сфере Римана с одним полюсом седьмого порядка и вещественными периодами. Оно возникает естественным образом при исследовании окрестности цикла Виттена $W_{5}$ в комбинаторной модели, основанной на квадратичных дифференциалах Дженкинса-Штребеля пространств модулей $\mathcal{M}_{g, n}$. Пространство $\mathcal{Q}_{0}^{\mathbb{R}}\left([-3]^{2}\right)$ - это пространство модулей мероморфных квадратичных дифференциалов на сфере Римана с двумя полюсами порядка не выше третьего и вещественными периодами. Оно возникает при описании окрестности границы Концевича $W_{1,1}$ комбинаторной модели. Применение формализма тау-функций Бергмана к комбинаторной модели для аналитического вычисления циклов, пуанкаре-дуальных определенным комбинациям тавтологических классов, требует изучения специальных сечений расслоений окружностей над $\mathcal{Q}_{0}^{\mathbb{R}}(-7)$ и $\mathcal{Q}_{0}^{\mathbb{R}}\left([-3]^{2}\right)$; в случае пространства $\mathcal{Q}_{0}^{\mathbb{R}}(-7)$ сечение этого расслоения окружностей задается аргументом модулярного дискриминанта. Подробно изучаются пространства $\mathcal{Q}_{0}^{\mathbb{R}}(-7)$ и $\mathcal{Q}_{0}^{\mathbb{R}}\left([-3]^{2}\right)$, также называемые пространствами кривых Бутру, и соответствующие расслоения окружностей.
\end{abstract}

Ключевые слова: пространства модулей, квадратичные дифференциалы, кривые Бутру, тау-функции, дифференциалы Дженкинса-Штребеля, ленточные графы.

DOI: https://doi.org/10.4213/tmf9513

Исследование М. Бертолы частично поддержано Natural Sciences and Engineering Research Council of Canada (грант RGPIN-2016-06660). Исследование Д. Короткина частично поддержано Natural Sciences and Engineering Research Council of Canada (грант RGPIN/3827-2015), а также Alexander von Humboldt Stiftung и GNFM Gruppo Nazionale di Fisica Matematica. Оба автора поддержаны FQRNT (грант "Matrices Aléatoires, Processus Stochastiques et Systèmes Intégrables" (2013-PR-166790)). Д. Короткин благодарит International School of Advanced Studies, Триест, Италия, и Max-Planck Institute for Gravitational Physics (Albert Einstein Institute), Гольм, Германия, за гостеприимство и поддержку в процессе написания статьи.

${ }^{*}$ Concordia University, Department of Mathematics and Statistics, Québec, Canada.

E-mail: Dmitry.Korotkin@concordia.ca, Marco.Bertola@concordia.ca

${ }^{\dagger}$ International School for Advanced Studies (SISSA), Area of Mathematics, Trieste, Italy. E-mail: mbertola@sissa.it 


\section{1. МОТИВАЦИЯ ИССЛЕДОВАНИЙ И СВОДКА РЕЗУЛЬТАТОВ}

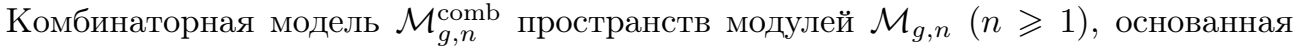
на дифференциалах Дженкинса-Штребеля (ДШ) [1]-[4], является весьма полезной при изучении геометрии пространства модулей. В частности, она была использована Концевичем [5], [6] для доказательства гипотезы Виттена [7] о числах пересечения $\psi$-классов.

В рамках этого подхода комбинаторная модель предсталяет собой CW-комплекс, в котором каждая клетка гомеоморфна симплексу, отвечающему ленточному графу, вложенному в риманову поверхность. Как было показано в работах [8]-[11], авторы которых следовали более ранней работе [12] (где была использована комбинаторная модель пространства $\mathcal{M}_{g, n}$, основанная на гиперболической геометрии) и статье [13], естественные циклы в комбинаторной модели можно интерпретировать как пуанкаре-дуальные определенным комбинациям классов Миллера-Мориты-Мамфорда $(\kappa$-классов). Эти естественные циклы состоят из клеток, соответствующих ленточным графам с фиксированной нечетной валентностью вершин: клетки наивысшей размерности отвечают трехвалентным ленточным графам.

Если ленточный граф содержит только одну вершину с валентностью, отличной от 3, то такие циклы называются циклами Виттена; если число таких невырожденных вершин больше единицы, то объединение соответствующих клеток называется циклом Концевича. Особую роль играют следующие два цикла. Первый их них цикл Виттена $W_{5}$, содержащий ленточные графы с одной пятивалентной вершиной и остальными вершинами валентности 3. Второй цикл является границей Концевича $W_{1,1}$ комбинаторной модели, содержащей ленточные графы, которые имеют две одновалентные вершины и остальные вершины валентности 3. Граница Концевича $W_{1,1}$ - это подмножество границы Делиня-Мамфорда пространства $\mathcal{M}_{g, n}$ (некоторые компоненты границы Делиня-Мамфорда сопоставляются одной точке в $\left.W_{1,1}\right)$. Вообще говоря, цикл Концевича-Виттена $W_{\left(k_{1}, \ldots, k_{r}\right)}$ содержит клетки, отвечающие графам Штребеля, у которых $r$ вершин имеют валентность $2 k_{j}+3\left(k_{j} \geqslant-1\right)$, а остальные вершины имеют валентность 3 (см. работу [8]).

Отправной точкой расчетов, связывающих комбинаторные циклы с $\kappa$-классами и их комбинациями, служит явное выражение для форм связности в расслоениях окружностей (или $U(1)$-расслоениях), отвечающих тавтологическим линейным расслоениям $\mathcal{L}_{k}$, ассоциированным с $k$-м проколом (см. детали в [5], [14], [8], [10], [11]).

Основной причиной, побудившей нас написать настоящую работу, является желание заложить аналитическую основу применения методов тау-функции Бергмана к комбинаторной модели $\mathcal{M}_{g, n}^{\text {comb }}$ пространства $\mathcal{M}_{g, n}$. Тау-функция Бергмана впервые появилась в теории изомонодромных деформаций [15] и фробениусовых многообразий [16] в контексте пространств Гурвица. Затем теория была расширена до пространств модулей абелевых [17] и квадратичных [18] дифференциалов над римановыми поверхностями. С геометрической точки зрения тау-функция Бергмана есть (голоморфное или мероморфное) сечение произведения линейного расслоения Ходжа и других естественных линейных расслоений над соответствующим пространством модулей. Поэтому в рамках голоморфного подхода анализ структуры ее сингулярностей позволяет получить различные соотношения в группах Пикара 
этих пространств модулей [19]-[21]. Прямое применение данной техники в контексте комбинаторной модели $\mathcal{M}_{g, n}$ на основе дифференциалов ДШ затруднительно из-за отсутствия естественной голоморфной структуры на клетках. Тау-функция Бергмана, надлежащим образом определенная на комбинаторной модели ДШ пространства $\mathcal{M}_{g, n}$, является только вещественной и аналитической в каждой ячейке; однако ее аргумент может использоваться для получения гладкого сечения расслоения окружностей. В свою очередь, изучение монодромии этих сечений позволяет найти циклы комбинаторной модели, пуанкаре-дуальные некоторым комбинациям стандартных тавтологических классов в пространстве $\mathcal{M}_{g, n}$ [22]. Реализация этой идеи требует изучения локального поведения тау-функции Бергмана и соответствующего ей расслоения окружностей в окрестности циклов $W_{5}$ и $W_{1,1}$.

Далее мы описываем окрестности циклов $W_{5}$ и $W_{1,1}$ в $\mathcal{M}_{g, n}^{\text {comb }}$, применяя соответствующую конформную спайку (conformal welding), которую эквивалентным образом можно интерпретировать с помощью так называемой конструкиии вклейки трубки (plumbing construction), используя плоскую структуру, вводящуюся на римановой поверхности дифференциалом ДШ.

Комбинаторные модели в окрестности циклов $W_{5}$ и $W_{1,1}$. Анализ окрестности цикла Виттена $W_{5}$ в пространстве $\mathcal{M}_{g, n}^{\text {comb }}$ показывает, что возникает вещественное сечение $\mathcal{Q}_{0}^{\mathbb{R}}(-7)$ (пространство кривых Бутру) пространства модулей $\mathcal{Q}_{0}(-7)$ мероморфных квадратичных дифференциалов на сфере Римана с одним полюсом седьмого порядка.

Пространство $\mathcal{Q}_{0}^{\mathbb{R}}(-7)$ возникает при изучении окрестности цикла $W_{5} \subset \mathcal{M}_{g, n}^{\text {comb }}$ следующим образом: клетки, образующие цикл $W_{5}$, получаются из клеток наивысшей размерности в пространстве $\mathcal{M}_{g, n}^{\text {comb }}$ путем вырождения двух ребер с ровно одной общей вершиной; это вырождение приводит к возникновению так называемой зоны вклейки трубки (как это показано ниже на рис. 6), которая разделяет компоненту $\mathbb{C P}^{1}$, содержащую три нуля дифференциала $Q$, и его полюс седьмой степени в нодальной точке. Тогда квадратичный дифференциал $Q$, возникающий на отделенной сфере Римана, принадлежит пространству $\mathcal{Q}_{0}^{\mathbb{R}}(-7)$.

Пространство $\mathcal{Q}_{0}^{\mathbb{R}}\left([-3]^{2}\right)$ естественным образом возникает в окрестности границы Концевича $W_{1,1}$ : оно является пространством модулей квадратичных дифференциалов на сфере Римана с двумя полюсами третьего порядка. Клетки, образующие $W_{1,1}$, получаются из клеток наивысшей размерности путем одновременного вырождения двух ребер с двумя общими вершинами. Такое вырождение приводит к возникновению двух зон вклейки трубки (как это показано ниже на рис. 10). Тогда квадратичный дифференциал $Q$, возникающий на отделяющейся сфере Римана, принадлежит пространству $\mathcal{Q}_{0}^{\mathbb{R}}\left([-3]^{2},[1]^{2}\right)$.

Оба пространства $\mathcal{Q}_{0}^{\mathbb{R}}(-7)$ и $\mathcal{Q}_{0}^{\mathbb{R}}\left([-3]^{2}\right)$ имеют вещественную размерность 2 . $\mathrm{K}$ нашему удивлению, мы не нашли в литературе полного описания этих двух элементарных пространств модулей; одна из целей настоящей статьи - заполнить этот пробел. Стратификация данных пространств, а также их комплексифицированные версии $\mathcal{Q}_{0}(-7)$ и $\mathcal{Q}_{0}\left([-3]^{2}\right)$ детально рассмотрены в п. 5.1, 6.1.

Пространство $\mathcal{Q}_{0}^{\mathbb{R}}(-7)$. Элемент общего вида комплексного пространства $\mathcal{Q}_{0}(-7)$ можно задать как квадратичный дифференциал

$$
Q=\left(x-x_{1}\right)\left(x-x_{2}\right)\left(x-x_{3}\right)(d x)^{2}, \quad x_{1}+x_{2}+x_{3}=0 .
$$


Локальные гомологические координаты определяются интегралами от $v=\sqrt{Q}$ по двум независимым гомологическим циклам на эллиптической кривой (каноническом накрытии) $\widehat{\mathcal{C}}$, заданной уравнением $v^{2}=Q$ (см. детали в п. 5.1). Вещественный слой $\mathcal{Q}_{0}^{\mathbb{R}}(-7)$ в пространстве $\mathcal{Q}_{0}(-7)$ задается условием, что все периоды дифференциала $v$ вещественны, и тогда пространство $\mathcal{Q}_{0}^{\mathbb{R}}(-7)$ состоит из клеток вещественной размерности 2, клетки размерности 1 (где два нуля дифференциала $Q$ совпадают) и клетки нулевой размерности (когда $x_{1}=x_{2}=x_{3}=0$ ).

Из вещественности всех периодов дифференциала $v$ следует, что период $\sigma$ кривой $\widehat{\mathcal{C}}$ принадлежит одномерному подмножеству $\mathcal{R}_{1}$ модулярной кривой, показанной ниже на рис. 11a.

Пространство $\mathcal{Q}_{0}^{\mathbb{R}}\left(-7,[1]^{3}\right)$ расслаивается над множеством $\mathcal{R}_{1}$ со слоем $\mathbb{R}_{+}$. Бесконечно удаленная точка в множестве $\mathcal{R}_{1}$ отвечает пространству $\mathcal{Q}_{0}^{\mathbb{R}}(-7,1,2)$. Точка пересечения множества $\mathcal{R}_{1}$ с вещественной осью в плоскости $J$-инварианта (см. рис. 11б) соответствует $J \simeq 940.34$, и отвечает кривой Бутру-Кричевера [23]-[25]. Она является единственной кривой Бутру в $\mathcal{Q}_{0}^{\mathbb{R}}\left(-7,[1]^{3}\right)$, имеющей вещественную инволюцию $\star$, которая оставляет инвариантным дифференциал ДШ: $\overline{Q\left(x^{\star}\right)}=Q(x)$.

Нули $x_{1}, x_{2}$ и $x_{3}$ связаны двумя горизонтальными (т. е. параллельными вещественной прямой в плоскости координаты $\left.z(x)=\int^{x} v\right)$ геодезическими в метрике $|Q|$. Мы всегда будем обозначать символом $x_{2}$ центральный ноль, который соединен геодезическими с двумя другими нулями. Оставшиеся нули $x_{1}$ и $x_{3}$ можно определить так, что при обходе начала координат в положительном направлении точка $x_{3}$ проходится после $x_{2}$, а точка $x_{1}$ - после $x_{3}$. Длины геодезических равны $A=\left|\int_{x_{1}}^{x_{2}} \sqrt{Q}\right|$, $B=\left|\int_{x_{2}}^{x_{3}} \sqrt{Q}\right|$. Длины $(A, B)$ задают отображение пространства $\mathcal{Q}_{0}^{\mathbb{R}}\left(-7,[1]^{3}\right)$ в $\mathbb{R}_{+}^{2}$.

Дискриминантное расслоение окружностей на $\mathcal{Q}_{0}^{\mathbb{R}}(-7)$. Рассмотрим сечение $U(1)$-расслоения над $\mathcal{Q}_{0}^{\mathbb{R}}\left([1]^{3},-7\right)$, заданное аргументом модулярного дискриминанта, $\phi_{5}=\operatorname{Arg} \Delta_{5}$, где

$$
\Delta_{5}=\left[\left(x_{1}-x_{2}\right)\left(x_{3}-x_{1}\right)\left(x_{3}-x_{2}\right)\right]^{2} .
$$

Дискриминант $\Delta_{5}$ равен нулю на границе страта $\mathcal{Q}_{0}^{\mathbb{R}}\left([1]^{3},-7\right)$. Однако его аргумент $\phi_{5}$ имеет корректно определенные пределы на лучах $A=0, B=0$ (т. е. при приближении к границе пространства модулей с двух разных сторон), причем эти пределы не зависят от точки на границе. Доказанная ниже теорема 2 показывает, что между $A=0$ и $B=0$ значение $\phi_{5}$ увеличивается на $\pi / 5$; этот результат технически нетривиален. Следовательно, величина $\pi / 5$ является монодромией аргумента $\phi_{5}$ при обходе клетки нулевой размерности (соответствующей дифференциалу $\left.Q=x^{3} d x^{2}\right)$ в пространстве $\mathcal{Q}_{0}^{\mathbb{R}}(-7)$.

Пространство $\mathcal{Q}_{0}^{\mathbb{R}}\left([-3]^{2}\right)$. Комплексное пространство модулей $\mathcal{Q}_{0}\left([-3]^{2}\right)$ состоит из квадратичных дифференциалов $Q$ на сфере Римана с двумя полюсами третьей степени. Общий дифференциал такого типа имеет вид

$$
Q=\frac{\left(x-x_{1}\right)\left(x-x_{2}\right)}{x^{3}}(d x)^{2}, \quad x_{1} \neq x_{2} \quad x_{1} \neq 0 \neq x_{2} .
$$

Интегралы от $\sqrt{Q}$ по путям в двух произвольных независимых гомологических классах канонического накрытия $y^{2}=x\left(x-x_{1}\right)\left(x-x_{2}\right)$ являются локальными координатами на $\mathcal{Q}_{0}\left([-3]^{2}\right)$. Пространство $\mathcal{Q}_{0}^{\mathbb{R}}\left([-3]^{2}\right)$ задается условием, что все периоды 
дифференциала $v=\sqrt{Q}$ вещественны; оно расслаивается над множеством $\mathcal{R}_{-1,-1}$ вещественной размерности 1 , лежащим в модулярной кривой (см. ниже рис. 15 , левая часть), со слоем $\mathbb{R}_{+}$.

Бесконечно удаленная точка в $\mathcal{R}_{-1,-1}$ отвечает пространству $\mathcal{Q}_{0}^{\mathbb{R}}\left(2,[-3]^{2}\right)$. Множество $\mathcal{R}_{-1,-1}$ пересекает вещественную ось $J$-инварианта при $J \simeq\{-1690,586,7791\}$ (см. рис. 15 , правая часть). Точки $J \simeq-1690$ и $J \simeq 586$ соответствуют кривым с вещественной инволюцией, но дифференциал $Q$ неинвариантен относительно этой инволюции. Точка $J \simeq 7791\left(x_{1} \simeq 1.8037, x_{2} \simeq-0.3797\right)$ отвечает кривой, обладающей вещественной инволюцией (действующей как стандартное комплексное сопряжение) $\overline{Q\left(x^{\star}\right)}=Q(x)$. Она является естественным аналогом кривой Бутру-Кричевера в пространстве $\mathcal{Q}_{0}^{\mathbb{R}}\left([-3]^{2}\right)$.

Один из нулей, который мы обозначим через $x_{1}$, связан бесконечными горизонтальными геодезическими в метрике $|Q|$ с полюсом $x=0$, а другой ноль $x_{2}-$ с полюсом $x=\infty$. Нули $x_{1}$ и $x_{2}$ соединены двумя горизонтальными геодезическими, которые мы обозначим через $e_{1}$ и $e_{2}$. Они определяются следующим образом: при достижении нуля $x_{1}$ вдоль горизонтальной геодезической, исходящей из $x=0$, при повороте направо мы следуем по геодезической $e_{1}$, а при повороте налево - по геодезической $e_{2}$ (см. ниже рис. 7). Длины геодезических $e_{1}$ и $e_{2}$ мы будем обозначать через $A$ и $B$. Длины $(A, B)$ задают отображение пространства $\mathcal{Q}_{0}^{\mathbb{R}}\left([1]^{2},[-3]^{2}\right)$ в $\mathbb{R}_{+}^{2}$.

Дискриминантное расслоение окружностей над $\mathcal{Q}_{0}^{\mathbb{R}}\left([-3]^{2}\right)$. Сечения $\phi_{1,1}^{ \pm}$ двух естественных $U(1)$-расслоений над $\mathcal{Q}_{0}^{\mathbb{R}}\left([1]^{2},[-3]^{2}\right)$ задаются следующими выражениями:

$$
\phi_{1,1}^{ \pm}=\operatorname{Arg} \Delta_{1,1}^{ \pm}, \quad \Delta_{1,1}^{+}:=x_{1}^{6} x_{2}^{6}\left(x_{1}-x_{2}\right)^{2}, \quad \Delta_{1,1}^{-}:=x_{1}^{6} x_{2}^{6}\left(x_{1}-x_{2}\right)^{26} .
$$

Анализ, представленный в разделе 6 , показывает, что между лучом $A=0$ и лучом $B=0$ в пространстве $\mathcal{Q}_{0}^{\mathbb{R}}\left([-3]^{2}\right)$ (т. е. при приближении к границе с двух разных сторон) величины $\phi_{1,1}^{+}$и $\phi_{1,1}^{-}$возрастают на $13 \pi$ и $25 \pi$ соответственно. Следовательно, монодромии величин $\phi_{1,1}^{+}$и $\phi_{1,1}^{-}$при обходе по простой, замкнутой, нестягиваемой петле в $\mathcal{Q}_{0}^{\mathbb{R}}\left([-3]^{2}\right)$ равны $13 \pi$ и $25 \pi$ соответственно (см. теорему 2 ).

Подводя итог, перечислим основные результаты статьи. Во-первых, мы показываем, что пространства модулей $\mathcal{Q}_{0}^{\mathbb{R}}(-7)$ и $\mathcal{Q}_{0}^{\mathbb{R}}\left([-3]^{2}\right)$ возникают из графа Штребеля общего вида в результате вырождения смежных ребер. Во-вторых, мы аналитически исследуем пространства $\mathcal{Q}_{0}^{\mathbb{R}}(-7)$ и $\mathcal{Q}_{0}^{\mathbb{R}}\left([-3]^{2}\right)$ и естественные расслоения окружностей над ними. Эти результаты обеспечивают необходимые аналитические инструменты для применения формализма тау-функции Бергмана при описании различных тавтологических классов в комбинаторной модели пространства $\mathcal{M}_{g, n}[22]$.

Статья организована следующим образом. В разделе 2 мы напоминаем основные сведения о комбинаторной модели $\mathcal{M}_{g, n}$, основанной на дифференциалах ДШ. В разделе 3 мы описываем окрестность цикла Виттена $W_{5}$ в комбинаторной модели и показываем, как в этом контексте возникает пространство модулей $\mathcal{Q}_{0}^{\mathbb{R}}(-7)$. В разделе 4 мы описываем окрестность границы Концевича $W_{1,1}$ пространства $\mathcal{M}_{g, n}^{\text {comb }}$ и показываем, каким образом возникает пространство $\mathcal{Q}_{0}^{\mathbb{R}}\left([-3]^{2}\right)$. В разделе 5 мы детально изучаем геометрию пространства $\mathcal{Q}_{0}^{\mathbb{R}}(-7)$ и вычисляем монодромию аргумента аргумент модулярного дискриминанта $\Delta_{5}$ на нем. Наконец, в разделе 6 мы изучаем геометрию пространства $\mathcal{Q}_{0}^{\mathbb{R}}\left([-3]^{2}\right)$ и вычисляем монодромии аргументов $\Delta_{1,1}^{ \pm}$в этом пространстве. 


\section{2. КОМБИНАТОРНАЯ МОДЕЛЬ ПРОСТРАНСТВА $\mathcal{M}_{g, n}$ И ДИФФЕРЕНЦИАЛЫ ДШ}

Вкратце напомним основные компоненты комбинаторной модели пространств модулей римановых поверхностей, основанной на дифференциалах ДШ.

Обозначим пространство модулей римановой поверхности рода $g$ с $n$ отмеченными точками через $\mathcal{M}_{g, n}$, а его компактификацию Делиня-Мамфорда - через $\overline{\mathcal{M}}_{g, n}$. Пусть $\mathcal{C}$ - риманова поверхность рода $g$ и $Q$ - мероморфный квадратичный дифференциал с полюсами второго порядка в точках $y_{1}, \ldots, y_{n}$. Обозначим нули дифференциала $Q$ через $x_{1}, \ldots, x_{m}$, а их кратности - через $\mathbf{d}=\left(d_{1}, \ldots, d_{m}\right)$; при этом $\sum_{i=1}^{m} d_{i}=4 g-4+2 n$. Пусть $\mathcal{Q}_{g, n}^{\mathrm{d}}-$ пространство модулей таких квадратичных дифференциалов; его размерность равна $2 g-2+n+m$. Для страта наивысшей размерности, когда все нули простые и $m=4 g-4+2 n$, эта размерность равна $6 g-6+3 n$.

Каноническое накрытие. Уравнение $v^{2}=Q$ в $T^{*} \mathcal{C}$ определяет двулистное накрытие $\widehat{\mathcal{C}}$ поверхности $\mathcal{C}$, в теории Тейхмюллера называющееся каноническим накрытием (в теории систем Хитчина $\widehat{\mathcal{C}}$ известно как спектральное накрытие, а в суперсимметричной теории Янга-Миллса его называют кривой Зайберга-Виттена). Точки ветвления этого накрытия совпадают с полюсами нечетного порядка дифференциала $Q$, их количество мы обозначаем через $m_{\text {odd }}$, при этом род канонического накрытия равен $\hat{g}=2 g+m_{\text {odd }} / 2-1$. В случае общего дифференциала $Q$, который имеет $4 g-4+2 n$ простых нулей, $\hat{g}=4 g-3+n$. Удобно ввести обозначение

$$
g_{-}=\hat{g}-g=g+\frac{m_{\text {odd }}}{2}-1
$$

Обозначим через $\mu$ голоморфную инволюцию, переставляющую листы накрытия $\widehat{\mathcal{C}}$. Абелев дифференциал $v$ третьего рода на $\widehat{\mathcal{C}}$ имеет $2 n$ простых полюсов в точ$\operatorname{kax}\left\{y_{i}, y_{i}^{\mu}\right\}$ (слегка злоупотребляя обозначениями, мы будем использовать символ $y_{i}$ для координаты полюса как на $\mathcal{C}$, так и на $\widehat{\mathcal{C}}$ ). Рассмотрим разложение пространства $H_{1}\left(\widehat{\mathcal{C}} \backslash\left\{y_{i}, y_{i}^{\mu}\right\}_{i=1}^{n}, \mathbb{R}\right)$ (его размерность равна $2 \hat{g}+2 n-1$ ) в прямую сумму четного и нечетного подпространств:

$$
H_{+} \oplus H_{-}, \quad \operatorname{dim} H_{+}=2 g+n-1, \quad \operatorname{dim} H_{-}=2 g_{-}+n .
$$

Заметим, что $\operatorname{dim} H_{-}=\operatorname{dim} \mathcal{Q}_{g, n}^{\mathbf{d}}$. Следовательно, в пространстве $\mathcal{Q}_{g, n}^{\mathbf{d}}$ можно ввести систему локальных координат, которые называются гомологическими координатами, выбирая в $H_{-}$независимые циклы $s_{i}, i=1, \ldots, \operatorname{dim} H_{-}$, и интегрируя по ним дифференциал $v$ :

$$
\mathcal{P}_{i}=\int_{s_{i}} v .
$$

Следующее определение дифференциала ДШ эквивалентно стандартному [2].

ОПРЕДЕЛЕНИЕ. Квадратичный дифференциал $Q$ называется дифференциалом ДШ, если все гомологические координаты $\mathcal{P}_{i}$ вещественны.

Из вещественности всех координат $\mathcal{P}_{i}$ следует, что бивычеты дифференциала $Q$ в полюсах $y_{j}$ являются вещественными и отрицательными, т. е. существуют такие 
числа $p_{j} \in \mathbb{R}_{+}$, что для любой локальной координаты $\zeta$ в окрестности точки $y_{j}$

$$
Q(\zeta)=-\frac{\left(p_{j} / 2 \pi\right)^{2}}{\zeta^{2}}(1+\mathcal{O}(\zeta))(d \zeta)^{2}
$$

Из результатов ДШ следует, что для заданных $p_{i} \in \mathbb{R}_{+}$существует единственный дифференциал $Q$ на заданной римановой поверхности с $n$ отмеченными точками, все периоды $\int_{s_{i}} v$ которого вещественны при $s_{i} \in H_{-}$(комбинация циклов вокруг $z_{i}$ также образует часть подпространства $H_{-}$; вещественность периодов дифференциала $v$ в окрестности этих циклов гарантирована формой бивычетов в (2.3)). Это утверждение обеспечивает возможность комбинаторного описания пространства $\mathcal{M}_{g, n}$.

Для каждого заданного вектора $\mathbf{p}=\left(p_{1}, \ldots, p_{n}\right) \in \mathbb{R}_{+}^{n}$ можно построить комбинаторную модель пространства модулей $\mathcal{M}_{g, n}$, которую мы будем обозначать через $\mathcal{M}_{g, n}[\mathbf{p}]$. А именно, рассмотрим для каждой римановой поверхности $\mathcal{C}$ рода $g$ с $n$ отмеченными точками $z_{1}, \ldots, z_{n}$ единственный дифференциал ДШ $Q$, сингулярная часть которого в точках $z_{i}$ имеет вид, заданный в $(2.3)$.

Страт $\mathcal{M}_{g, n}^{\mathbf{d}}[\mathbf{p}]$ в $\mathcal{M}_{g, n}[\mathbf{p}]$ состоит из римановых поверхностей с проколами, таких что дифференциал ДШ $Q$ имеет нули с кратностями $\mathbf{d}=\left(d_{1}, \ldots, d_{m}\right)$. Вещественная размерность $\mathcal{M}_{g, n}^{\mathbf{d}}[\mathbf{p}]$ задается формулой

$$
\operatorname{dim}_{\mathbb{R}} \mathcal{M}_{g, n}^{\mathbf{d}}[\mathbf{p}]=2 g-2+m .
$$

Максимальный страт соответствует дифференциалам ДШ с простыми нулями и имеет вещественную размерность $6 g-6+2 n$ (в этом случае $m=4 g-4+2 n$ ), которая совпадает с вещественной размерностью пространства $\mathcal{M}_{g, n}$.

Ориентированные горизонтальные траектории 1-формы $v$ на кривой $\widehat{\mathcal{C}}$ (которые проецируются в неориентированные горизонтальные траектории дифференциала $Q$ на римановой поверхности $\mathcal{C}$ ) соединяют нули $x_{i}$. Эти горизонтальные траектории на $\mathcal{C}$ образуют ребра вложенного графа (который также называется ленточный граф) $\Gamma$ на $\mathcal{C}$ с вершинами в точках $x_{1}, \ldots, x_{m}$, которые имеют валентности $d_{1}+2, \ldots, d_{m}+2$ соответственно. Таким образом, страт наивысшей размерности $6 g-6+2 n$ отвечает ленточному графу на римановой поверхности $\mathcal{C}$ с трехвалентными вершинами.

Длина $\ell_{e}$ ребра $e$, соединяющего вершины $v_{1}, v_{2}$, равна абсолютной величине интеграла от $v$ по горизонтальной траектории, которая соединяет вершины. В свою очередь, с точностью до множителя $1 / 2$ эта длина совпадает с интегралом от $v$ по целочисленным циклам в $H_{-}$, состоящим из траектории $e$ на одном листе проекции $\widehat{\mathcal{C}} \rightarrow \mathcal{C}$ и такой же траектории, но идущей в противоположном направлении на другом листе. Фиксируя вектор $\mathbf{p} \in \mathbb{R}_{+}^{n}$ мы налагаем $n$ линейных условий на длины ребер: для $j$-й грани $f_{j}, j=1, \ldots, n$, мы имеем $\sum_{e \in \partial f_{j}} \ell_{e}=p_{j}$.

Простой пример ленточного графа на римановой поверхности рода 1 с одной отмеченной точкой показан на рис. 1 . В плоской метрике $|Q|$ на $\mathcal{C}$ окрестность полюса $y_{i}$ представляет собой бесконечный цилиндр периметра $p_{i}$.

Объединение всех стратов $\mathcal{M}_{g, n}^{\mathbf{d}}[\mathbf{p}]$ при фиксированном $\mathbf{p} \in \mathbb{R}_{+}^{n}$ образует комбинаторную модель $\mathcal{M}_{g, n}[\mathbf{p}]$ пространства $\mathcal{M}_{g, n}$, которая с точки зрения теории множеств изоморфна $\mathcal{M}_{g, n}$. 

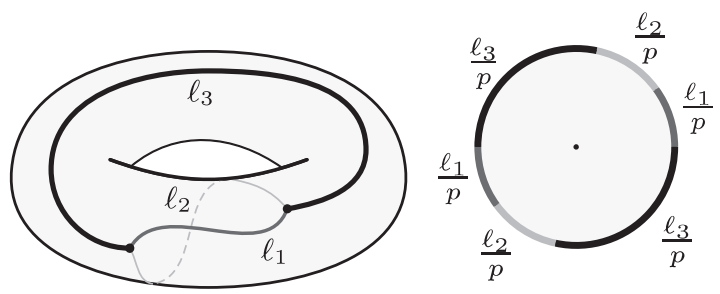

Рис. 1. Ленточный граф на римановой поверхности рода 1 , представляющий точку в пространстве $\mathcal{M}_{1,1}[p]$. Два простых нуля $x_{1}$ и $x_{2}$ дифференциала $Q$ связаны тремя ребрами, длины которых суть $\ell_{1}, \ell_{2}$ и $\ell_{3}$. Ленточный граф имеет единственную грань с периметром $p=2\left(\ell_{1}+\ell_{2}+\ell_{3}\right)$. Длины $\ell_{1}$ и $\ell_{2}$ можно использовать как координаты в пространстве $\mathcal{M}_{1,1}[p]$.

Компактификация $\overline{\mathcal{M}}_{g, n}[\mathbf{p}]$ данной комбинаторной модели строится путем добавления к $\mathcal{M}_{g, n}[\mathbf{p}]$ так называемой границы Концевича: страт $W_{1,1}$ границы Концевича, имеющий вещественную коразмерность 2 , соответствует ленточным графам с ровно двумя одновалентными вершинам (т. е. двумя простыми полюсами дифференциала ДШ $Q$ ), в то время как валентность остальных вершин остается равной 3. В пространстве $\mathcal{M}_{g, n}$ граница Концевича “меньше", чем граница Делиня-Мамфорда, поскольку комбинаторная модель ДШ неприменима для римановых поверхностей без проколов.

Каждой грани $F_{j}$ ленточного графа $\Gamma$ (грань $F_{j}$ содержит полюс $y_{j}$ ) можно сопоставить единичный диск с помощью отображения

$$
w_{j}(x)=\exp \left(\frac{2 \pi i}{p_{j}} \int_{x_{j}}^{x} v\right)
$$

где $x_{j}$ - произвольная вершина в грани $F_{j}$. При отображении $(2.5)$ точке $y_{j}$ сопоставляется начало координат, а точке $x_{j}$ сопоставляется 1 . Всегда можно выбрать ветвь дифференциала $v=\sqrt{Q}$, для которой вычет в $y_{j}$ равен $p_{j} / 2 \pi i$. На грани $F_{j}$ плоская метрика $|Q|=|v|^{2}$ римановой поверхности $\mathcal{C}$ записывается как

$$
|Q|=\frac{p_{j}^{2}}{4 \pi^{2}}\left|\frac{d w_{j}}{w_{j}}\right|^{2} .
$$

В комбинаторной модели $\mathcal{M}_{1,1}^{\mathrm{comb}}[p]$, показанной на рис. 1 , ленточный граф, соответствующий страту наибольшей размерности, имеет только одну грань; эта грань может быть отображена в единичный диск. Условие для длин в этом случае имеет вид $2\left(\ell_{1}+\ell_{2}+\ell_{3}\right)=p$. Пример ленточного графа на римановой поверхности более высокого рода показан на рис. 2.

В обратную сторону, из ленточного графа с заданными длинами всех ребер можно построить полиэдральную риманову поверхность (т. е. риманову поверхность с плоской метрикой и с коническими особенностями) с помощью так называемой конформной спайки [2].

Все страты комбинаторной модели $\mathcal{M}_{g, n}[\mathbf{p}]$ можно получить из страта $W$ наибольшей размерности (соответствующего ленточным графам, у которых все вершины 


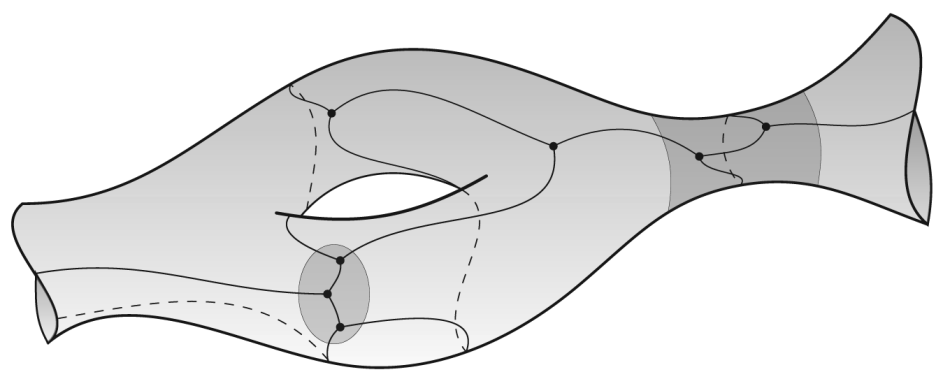

Рис. 2. Типичный вложенный граф Штребеля; в двух областях более темного цвета вырождение ребер приводит либо к клетке $W_{5}$ (левая область), либо к клетке $W_{1,1}$ (правая область).

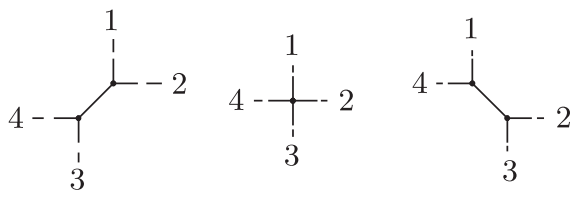

Рис. 3. Движение Уайтхеда.

трехвалентные) путем вырождения одного или нескольких ребер. Различные компоненты страта $W$ склеиваются вдоль границ (вещественной) коразмерности 1 , которые соответствуют ленточным графам с одной четырехвалентной вершиной и всеми остальными вершинами валентности 3 (т. е. дифференциал ДШ, соответствующий такой границе, имеет один двойной ноль, а другие нули простые). Процедура пересечения границы при переходе из одной ячейки слоя $W$ в другую (иногда таким способом можно попасть в одну и ту же ячейку, тогда ячейка граничит сама с собой) описывается так называемым движением Уайтхеда, показанным на рис. 3.

Наша цель состоит в том, чтобы подробно изучить вырождение двух ребер, имеющих одну общую вершину. В результате в зависимости от геометрии получаются либо клетки, образующие цикл Виттена $W_{5}$ (соответствующие ленточные графы имеют одну пятивалентную вершину, а другие вершины трехвалентные), либо клетки, образующие границу Концевича $W_{1,1}$ модели $\mathcal{M}_{g, n}[\mathbf{p}]$ (что соответствует ленточным графам с двумя одновалентными вершинам и всеми остальными вершинами валентности 3). Эти два типа вырождения показаны на рис. 2.

\section{3. ЛОКАЛЬНАЯ МОДЕЛЬ В ОКРЕСТНОСТИ ЦИКЛА ВИТТЕНА $W_{5}$ : ПРОСТРАНСТВО $\mathcal{Q}_{0}^{\mathbb{R}}(-7)$}

Клетки цикла $W_{5}$ в комбинаторной модели $\mathcal{M}_{g, n}[\mathbf{p}]$ (во всех конструкциях, приведенных ниже, вектор р считается фиксированным) отвечают ленточным графам, все вершины которых имеют валентность 3, за исключением одной вершины с валентностью 5. Здесь мы рассматриваем риманову поверхность $\mathcal{C}$ с $n$ проколами как элемент комбинаторной модели $\mathcal{M}_{g, n}[\mathbf{p}]$, следовательно, $\mathcal{C}$ единственным образом определяет дифференциал ДШ $Q$. 
Точку цикла $W_{5}$ можно получить из трехвалентного ленточного графа путем вырождения двух ребер, имеющих одну общую вершину, и наоборот, любая риманова поверхность из окрестности поверхности $\mathcal{C}$ представляет собой двухпараметрическую деформацию поверхности $\mathcal{C}$ (с вещественными параметрами) в $W$. Далее мы покажем, как можно описать эту деформацию с помощью плоской перестройки римановой поверхности $\mathcal{C}$ и сферы Римана, оснащенной специальным квадратичным дифференциалом с вещественной нормировкой. Эта плоская перестройка также дает дифференциал ДШ в явном виде и ленточный граф на деформированной римановой поверхности. Понять, что такое плоская перестройка, можно также, используя так называемую конструкцию вклейки трубки, которая соединяет $\mathcal{C}$ со сферой Римана, наделенной соответствующей плоской метрикой.

Обозначим ноль третьего порядка дифференциала ДШ на $\mathcal{C}$ через $\tilde{x}$; также введем обозначение $\Gamma$ для ленточного графа, соответствующего $\mathcal{C}$. Пусть $\zeta$ - выделенный локальный параметр на $\mathcal{C}$ в окрестности точки $\tilde{x}: \quad \zeta(x)=\left[\frac{5}{2} \int_{\tilde{x}}^{x} v\right]^{2 / 5}$ (функция $\zeta(x)$ определена с точностью до корня пятой степени из единицы). При этом $Q(x)$ можно записать в терминах $\zeta$ как $Q(\zeta)=\zeta^{3}(d \zeta)^{2}$. Локальные координаты на каноническом накрытии $\widehat{\mathcal{C}}$ в окрестности точки $x_{1}$ задаются как $\zeta^{1 / 2}$.

Также (и на $\mathcal{C}$, и на $\widehat{\mathcal{C}}$ ) введем плоскую координату $z(x)=\int_{\tilde{x}}^{x} v$; в окрестности точки $\tilde{x}$ плоская координата выражается через локальную координату $\zeta$ следующим образом: $z=\frac{2}{5} \zeta^{5 / 2}$. Мы имеем $Q(x)=(d z(x))^{2}$ на $\mathcal{C}$, а на $\widehat{\mathcal{C}}$ получаем $v=d z$. Метрика $|Q(x)|$ имеет коническую точку в $\tilde{x}$ с углом $5 \pi$, в то время как в остальных вершинах угол равен $3 \pi$.

\section{1. Разрешение пятивалентной вершины путем плоской перестройки.}

Из вершины $x_{1}$ исходят пять ребер ленточного графа $Г$ в направлениях, заданных углами $2 \pi k / 5, k=0,1, \ldots, 4$, в плоскости переменной $\zeta$. Обозначим их длины (начав с ребра, идущего вдоль положительной части действительной оси) через $\ell_{1}, \ldots, \ell_{5}$. Построим в явном виде деформацию $\mathcal{C}^{\alpha, \beta}$ поверхности $\mathcal{C}$, содержащую два вещественных (малых) параметра $\alpha$ и $\beta$, изменяя длины следующим образом:

$$
\left(\ell_{1}, \ldots, \ell_{5}\right) \rightarrow\left(\ell_{1}-(\alpha+\beta), \ell_{2}+\beta, \ell_{3}-\beta, \ell_{4}-\alpha, \ell_{5}+\alpha\right)
$$

Разумеется, (циклическая) нумерация пяти ребер выбирается любым из пяти возможных способов. Тройной ноль $\tilde{x}$ дифференциала $Q$ на $\mathcal{C}$ расщепляется на три простых нуля $\left(x_{1}, x_{2}, x_{3}\right)$ дифференциала ДШ $Q^{\alpha, \beta}$ на $\mathcal{C}^{\alpha, \beta}$. Предположим, что новые ребра длины $\ell_{1}-(\alpha+\beta)$ и $\ell_{2}+\beta$ встречаются в $x_{1}$; вершина $x_{1}$ соединена с $x_{2}$ ребром длины $\alpha$, вершина $x_{2}$ - конечная точка ребра длины $\ell_{2}+\beta$, эта вершина также связана с $x_{3}$ ребром длины $\beta$; ребра с длинами $\ell_{4}-\alpha$ и $\ell_{5}+\alpha$ встречаются друг с другом в $x_{3}$. Получающаяся в результате конфигурация показана на рис. 4 .

Зафиксируем некоторый радиус $R$, считая, что $R<\ell_{i}$ для $i=1, \ldots, 5$. Рассмотрим диск $\mathcal{D}_{R}$ на поверхности $\mathcal{C}$, заданный условием $|z(x)| \leqslant R$ (см. рис. 4); в терминах выделенной локальной координаты $\zeta$ диск $\mathcal{D}_{R}$ задается неравенством $|\zeta| \leqslant(5 R / 2)^{2 / 5}$. Пять ребер ленточного графа $\Gamma$ внутри диска $\mathcal{D}_{R}$ образуют пять отрезков $\left[0,(5 R / 2)^{2 / 5}\right] e^{2 i \pi k / 5}, k=0, \ldots, 4$, в плоскости переменной $\zeta$. Периметр диска $\mathcal{D}_{R}$ в плоской метрике $|Q|$ равен $5 \pi R$. 


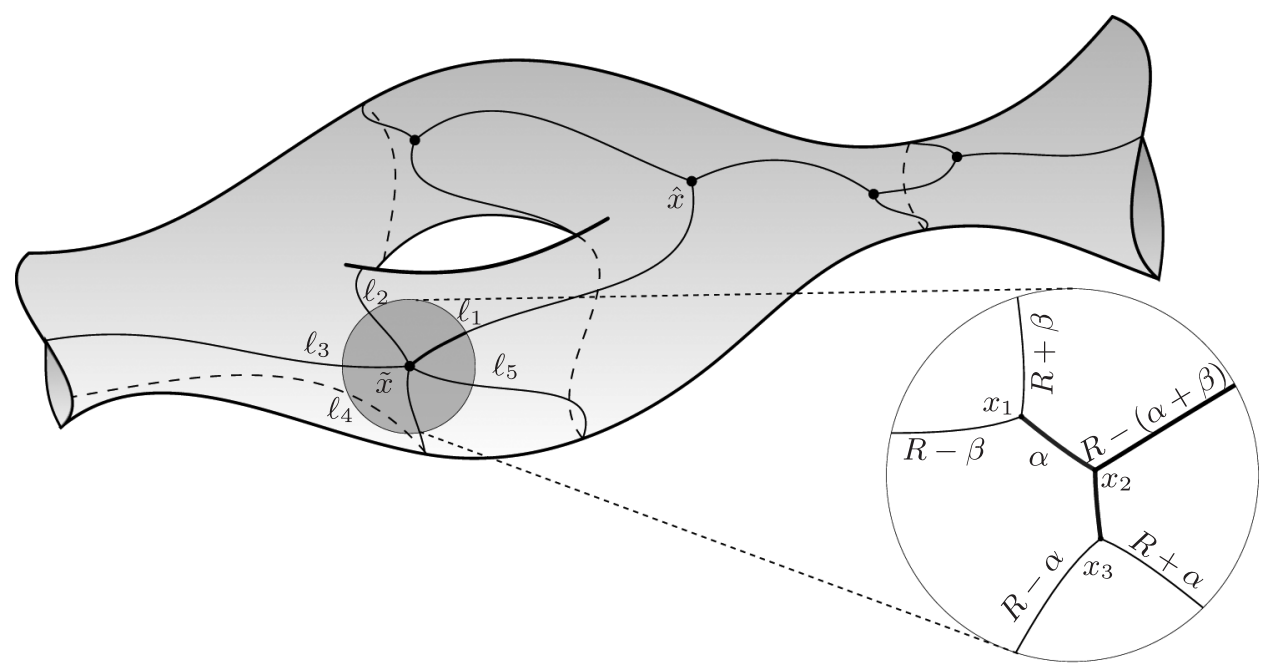

Рис. 4. Риманова поверхность $\mathcal{C}^{\alpha, \beta}$ получается заменой пятивалентной вершины тремя трехвалентными вершинами и двумя ребрами длины $\alpha$ и $\beta$ между ними при соответствующей корректировке длин ребер оригинального ленточного графа. Предел $\alpha, \beta \rightarrow 0$ воспроизводит исходную риманову поверхность. Пять дуг на границе (см. врезку) представляют собой пять полуокружностей радиуса $R$ в плоских координатах любой из метрик.

Теперь вырежем область $\mathcal{D}_{R}$ из римановой поверхности $\mathcal{C}$ и получим риманову поверхность с границей, эту поверхность обозначим через $\mathcal{C}_{R}$. Пусть $\widehat{\mathcal{C}}_{R}-$ каноническое накрытие $\widehat{\mathcal{C}}$, в котором диск $\mathcal{D}_{R}$ удален на обеих копиях поверхности $\mathcal{C}$. Предположим, что разрез, соединяющий тройной ноль $\tilde{x}$ с некоторым другим нулем на поверхности $\mathcal{C}$, идет вдоль положительной части вещественной оси координаты $\zeta$. Разрез можно выбрать пятью способами, и этот выбор определяет, как вклеивается диск $\mathcal{D}_{R}^{\alpha, \beta}$ (описанный ниже), давая, таким образом, пять различных семейств деформаций. Обозначим через $\mathcal{C}^{\alpha, \beta}$ одно из этих пяти семейств.

Рассмотрим элемент пространства модулей $\mathcal{Q}_{0}^{\mathbb{R}}[-7]$, такой что квадратичный дифференциал $Q_{0}$ на $\mathbb{C P}^{1}$ задается как

$$
Q_{0}(x)=\left(x-x_{1}\right)\left(x-x_{2}\right)\left(x-x_{3}\right)(d x)^{2}, \quad x_{1}+x_{2}+x_{3}=0 .
$$

Абелев дифференциал $v_{0}=\sqrt{Q_{0}}$ определен на каноническом накрытии $\widehat{\mathcal{C}_{0}}$ сферы Римана, т. е. является эллиптической кривой с точками ветвления в $x_{1}, x_{2}, x_{3}$ и $\infty$.

Предположим, что все периоды дифференциала $v_{0}$ на $\widehat{\mathcal{C}_{0}}$ вещественны. Тогда на $\mathbb{C P}^{1}$ существуют две горизонтальные траектории дифференциала $v_{0}$, которые соединяют точки $x_{1}, x_{2}$ и $x_{3}$. Предположим, что $x_{2}$ - центральный ноль, т. е. он соединен горизонтальными траекториями с $x_{1}$ и $x_{3}$. Проведем разрез на $\widehat{\mathcal{C}_{0}}$, чтобы соединить горизонтальными траекториями точку $x_{2}$ с $x_{1}, x_{3}$ и $\infty$. Предположим, что нумерация точек $x_{1}$ и $x_{3}$ введена так, что разрезы $\left[x_{2}, \infty\right),\left[x_{2}, x_{1}\right]$ и $\left[x_{2}, x_{3}\right]$, сходящиеся в точке $x_{2}$, расположены в указанном порядке, если их отсчитывать против 


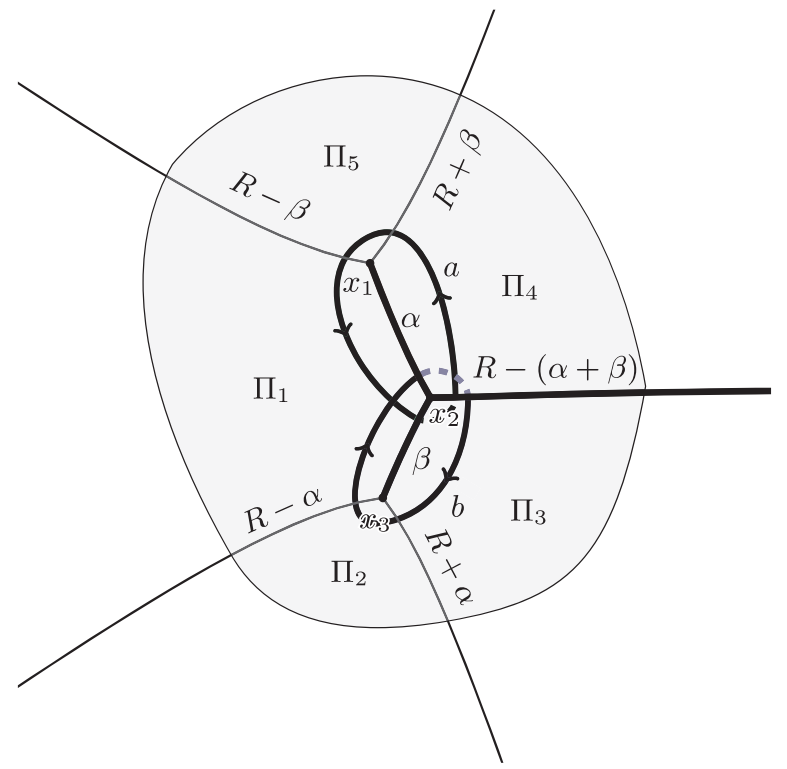

a

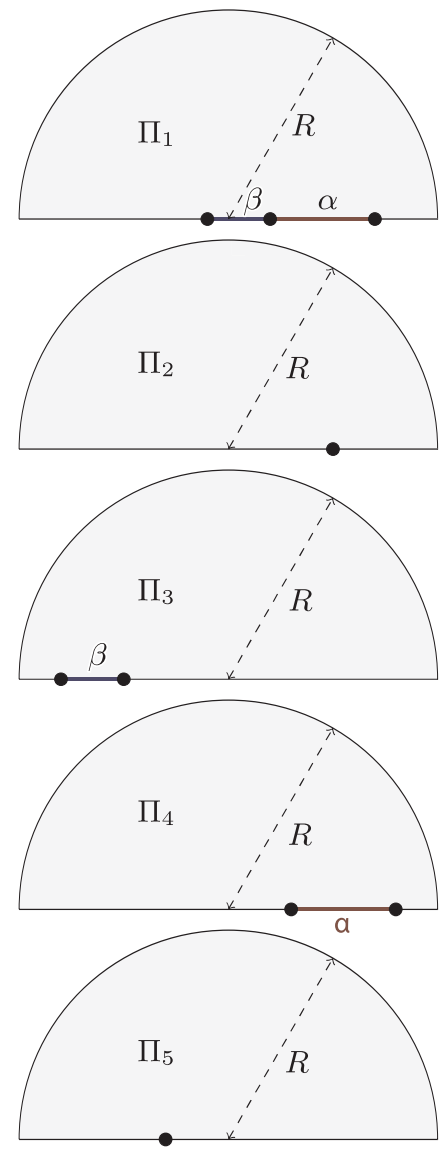

б

Рис. 5. Ленточный граф в пространстве $\mathbb{C P}^{1}$, отвечающий квадратичному дифференциалу $Q_{0}$, и геодезический диск радиуса $R$ в плоскости координаты $x$; помечены расстояния в метрике $\left|Q_{0}\right|$ вдоль траекторий (а). Пять полудисков в верхней полуплоскости отображаются с помощью плоской координаты (б).

часовой стрелки, как показано на рис. 5а (этот рисунок изображен в плоскости координаты $x)$.

Введем в $\widehat{\mathcal{C}}_{0}$ канонический базис циклов $(a, b)$ такой, что $a$-цикл окружает точки ветвления $x_{1}$ и $x_{2}$, а $b$-цикл окружает точки ветвления $x_{2}$ и $x_{3}$. Вещественные периоды дифференциала $v_{0}$ по циклам $a$ и $b$ выражаются через длины $\alpha$ и $\beta$ разрезов $\left[x_{2}, x_{1}\right]$ и $\left[x_{2}, x_{3}\right]$ следующим образом:

$$
\left|\int_{a} v_{0}\right|=2 \alpha, \quad\left|\int_{b} v_{0}\right|=2 \beta .
$$

Эти равенства задают точки ветвления $x_{i}$ с точностью до умножения всех $x_{i}$ на корень пятой степени из единицы (см. теорему 1). 
ЗАмечание 1. Основываясь на рис. 5, можно показать, что гомологические координаты $\int_{a} v_{0}, \int_{b} v_{0}$ имеют одинаковый знак. Для этого достаточно проверить знак плоской координаты, отсчитываемой от $x_{2}$, с учетом того, что три разреза сходятся в $x_{2}$. Следовательно, модуль в выражениях (3.3) можно не писать, если определить дифференциал $v_{0}$ так, что оба интеграла положительны.

Квадратичный дифференциал $Q_{0}$, имеющий на $\mathbb{C P}^{1}$ три простых нуля и один полюс седьмого порядка, является элементом пространства $\mathcal{Q}_{0}^{\mathbb{R}}\left([1]^{3},-7\right)$; это аналог дифференциала ДШ в случае, когда полюс имеет порядок выше двух.

Мы выбираем разрезы вдоль ребер $\left[x_{1}, x_{2}\right],\left[x_{3}, x_{2}\right]$ и $\left[x_{2}, \infty\right]$ (жирные линии на рис. 5a). Ребра ленточного графа $\Gamma_{0}$ делят плоскость переменной $x$ на пять областей, как показано на рис. 5а. Пять горизонтальных траекторий дифференциала $v_{0}$, которые соединяют $x_{1}, x_{2}$ и $x_{3} \mathrm{c} \infty$, уходят на $\infty$ вдоль лучей $\arg x=2 \pi k / 5$. В плоской метрике $\left|Q_{0}\right|$ на $\mathbb{C P}^{1}$ любая из этих пяти областей в терминах плоской координаты $z$ превращается в одну и ту же полуплоскость $\operatorname{Im} z>0$.

На каждом из пяти лучей, соединяющих бесконечно удаленную точку с точками $x_{i}$, вырежем отрезки, длины которых выберем как $R-\alpha-\beta, R+\beta, R-\beta, R-\alpha$ и $R+\alpha$ (если вести нумерацию лучей против часовой стрелки, начав с $\left[x_{2}, \infty\right)$ ). Затем в каждой области рассмотрим полукруг (в плоской метрике $\left|Q_{0}\right|$ ), который соединяет конечные точки выбранных отрезков (см. рис. 5б). Легко видеть, что диаметры всех этих пяти полукругов совпадают и равны $2 R$. Таким способом мы получаем пять областей, имеющих в плоской координате $z$ вид полудисков; обозначим их через $\Pi_{1}, \ldots, \Pi_{5}$, а их объединение - через $\mathcal{D}_{R}^{\alpha, \beta}$.

В каждой из областей $\Pi_{i}$ плоские координаты определяются с точностью до знака и сдвига, глобальная координата на всем $\mathcal{D}_{R}^{\alpha, \beta}$ задается следующим образом. Пусть начальная точка пути интегрирования есть $x_{2}$, выберем систему трех разрезов внутри области $\mathcal{D}_{R}^{\alpha, \beta}$, как показано на рис. 5а, тогда плоская координата на $\mathcal{D}_{R}^{\alpha, \beta}$ с тремя удаленными разрезами есть

$$
w(x)=\alpha+\beta+\int_{x_{2}}^{x} v_{0},
$$

где дифференциал $v_{0}$ должен быть определен так, чтобы образом области П 4 была верхняя полуплоскость переменной $z$. Нетрудно проверить, что в соответствии с ориентацией $a$ - и $b$-циклов на $\mathcal{C}_{0}$, показанной на рис. 5 а, значения координаты $w(x)$ на разных сторонах разреза $\left[x_{2}, \infty\right)$ отличаются знаком.

Обозначим каноническое двулистное накрытие области $\mathcal{D}_{R}^{\alpha, \beta}$ с разрезами вдоль горизонтальных траекторий $\left[x_{2}, x_{1}\right],\left[x_{2}, x_{3}\right]$ и $\left[x_{2}, \infty\right)$ через $\widehat{\mathcal{D}}_{R}^{\alpha, \beta}$.

Граница области $\mathcal{D}_{R}^{\alpha, \beta}$ в глобальной плоской координате представляет собой границу конуса углового размера $5 \pi$, а ее периметр равен $5 \pi R$. Положение точек, где исходящие ребра (в координате $z$ ) пересекают границу области $\mathcal{D}_{R}^{\alpha, \beta}$, совпадают с положением точек на $\mathcal{D}_{R}$. В частности, периметр области $\mathcal{D}_{R}^{\alpha, \beta}$ также равен $5 \pi R$. Следовательно, мы можем отождествить границу поверхности $\mathcal{C}_{R}$ и границу области $\mathcal{D}_{R}^{\alpha, \beta}$ (эта процедура в монографии [2] названа конформной спайкой), чтобы получить новую риманову поверхность $\mathcal{C}^{\alpha, \beta}$ вместе с ее каноническим накрытием $\widehat{\mathcal{C}}^{\alpha, \beta}$. 


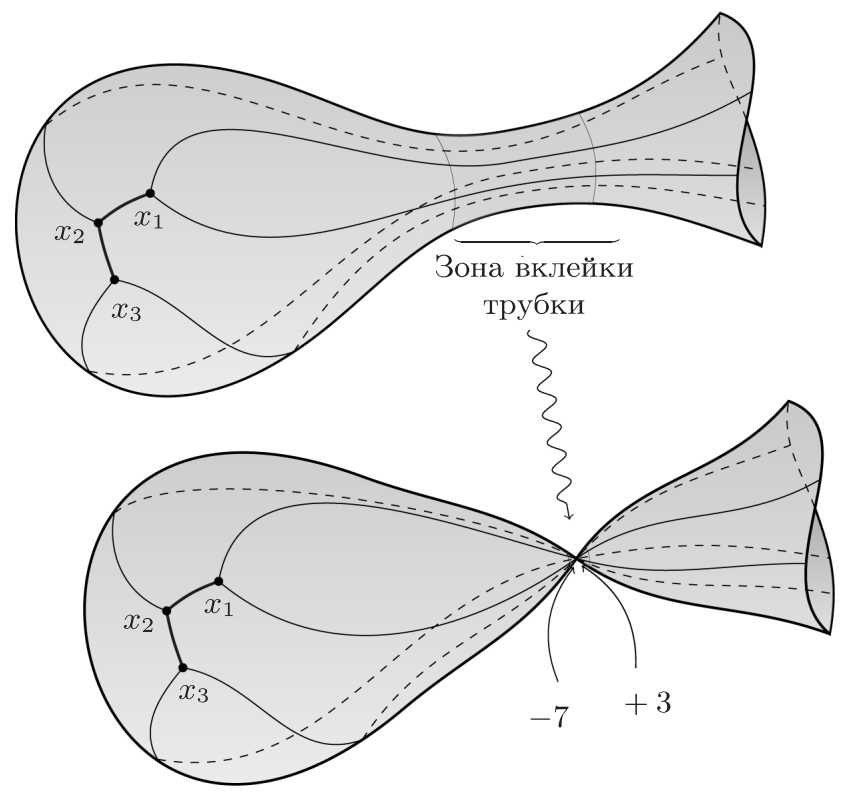

Рис. 6. Отделение сферы Римана с тремя простыми нулями и полюсом седьмого порядка в дифференциале $Q$ в окрестности цикла $W_{5}$.

Длины пяти ребер, изначально сходящихся в нуле третьего порядка на $\mathcal{C}$, в результате изменяются в соответствии с (3.1). Заметим, что эта деформация сохраняет периметры всех граней ленточного графа, поэтому риманова поверхность $\mathcal{C}^{\alpha, \beta}$ и соответствующий ленточный граф принадлежат к той же комбинаторной модели $\mathcal{M}_{g, n}[\mathbf{p}]$, что и исходная поверхность $\mathcal{C}$.

Кроме того, результат конформной спайки не зависит от радиуса $R$ вырезанного диска, пока это радиус остается достаточно малым по сравнению с $\ell_{1}, \ldots, \ell_{5}$ и достаточно большим по сравнению с $\alpha$ и $\beta$.

3.2. Конструкция вклейки трубки. Для изучения предела $\alpha, \beta \rightarrow 0$ в рассмотренной выше схеме конформной спайки нужно преобразовать квадратичный дифференциал (3.2) на $\mathbb{C P}^{1}$. Представленная в данном пункте эквивалентная конструкция вклейки трубки позволяет сохранить $\mathcal{C}$ и $\mathcal{C}_{0}$ фиксированными.

Чтобы деформировать поверхность $\mathcal{C}$ в $\mathcal{C}^{\alpha, \beta}$ (и наоборот, чтобы изучить предел $\alpha, \beta \rightarrow 0$ для $\left.\mathcal{C}^{\alpha, \beta}\right)$, введем параметр трубки $t=\alpha+\beta$ и величины

$$
A=\frac{\alpha}{t}=\frac{\alpha}{\alpha+\beta}, \quad B=\frac{\beta}{t}=\frac{\beta}{\alpha+\beta}, \quad A+B=1 .
$$

Теперь вырежем из поверхности $\mathcal{C}$ диск $\mathcal{D}_{2 t}$ радиуса $R=2 t$ с центром в точке $x_{1}$. Рассмотрим квадратичный дифференциал $Q_{0}$ в виде (3.2), при этом абсолютные величины периодов соответствующего абелева дифференциала $v_{0}$ равны $2 A$ и $2 B$ (вместо $2 \alpha$ и $2 \beta$, как выше), так что длины конечных ребер соответствующего ленточного графа равны $A$ и $B$. 
Аналогично конструкциям предыдущего пункта вырежем из сферы Римана область $\mathcal{D}_{2 / t}^{A, B}$. Радиус дырки в $\mathcal{C} \backslash \mathcal{D}_{2 t}$ равен $2 t$, а радиус области $\mathcal{D}_{2 / t}^{A, B}$ равен $2 / t$. Таким образом, обозначив, как и выше, плоскую координату на $\mathcal{C}$ в окрестности $\tilde{x}$ через $z$, а плоскую координату вблизи границы области $\mathcal{D}_{2 / t}^{A, B}$ - через $w$, зададим границу уравнением $z / w=t^{2}$. В то же время выделенная координата на $\mathcal{C}$ в окрестности $\tilde{x}$ (в терминах плоской координаты $z=\int_{\tilde{x}}^{x} v$ ) есть $\zeta=\left(\frac{5}{2} z\right)^{2 / 5}$. На сфере Римана рассмотрим локальную координату, записывающуюся в терминах глобальной плоской координаты $w(x)=1+\int_{x_{2}}^{x} v_{0}$ (аналогичной $\left.(3.4)\right)$ как $\xi(x)=\left(\frac{5}{2} w(x)\right)^{-2 / 5}$.

Далее рассмотрим на множестве $\mathcal{C} \backslash \mathcal{D}_{2 t}$ малое кольцо $A_{\epsilon}$, заданное условием $2 t<|z(x)|<2 t+\epsilon$ (с координатой $\zeta$ в $A_{\epsilon}$ ). Тогда кольца $A_{\epsilon}$ и $A_{\epsilon}^{0}$, необходимые в стандартной конструкции вклейки трубки, задаются соотношением $\xi(x) \zeta(y)=t^{4 / 5}$, где $x \in A_{\epsilon}, y \in A_{\epsilon}^{0}$.

Подход, связанный с конструкцией вклейки трубки, проиллюстрирован на рис. 6 . Заметим следующее отличие нашего подхода от стандартной картины. Обычно параметр трубки является комплексным и может использоваться в качестве локальной координаты вблизи границы. В нашем подходе мы имеем два вещественных параметра деформации: один из них является параметром трубки, а второй есть отношение $B / A \in[0, \infty]$, которое определяет мероморфный дифференциал на приклеенной сфере Римана.

\section{4. ЛОКАЛЬНАЯ МОДЕЛЬ В ОКРЕСТНОСТИ ГРАНИЦЫ КОНЦЕВИЧА $W_{1,1}$ И ПРОСТРАНСТВО $\mathcal{Q}_{0}^{\mathbb{R}}\left([-3]^{2}\right)$}

Клетки главного страта $W_{1,1}$ границы Коцевича комбинаторной модели $\mathcal{M}_{g, n}[\mathbf{p}]$ отвечают ленточным графам, у которых все вершины техвалентные, кроме двух вершин с валентностью 1. Одновалентные вершины появляются в результате разрешения нодальной точки. Соответствующая риманова поверхность $\mathcal{C}$ представляет собой нодальную кривую, лежащую на границе Делиня-Мамфорда пространства $\mathcal{M}_{g, n}$. Дифференциал ДШ $Q$ на $\mathcal{C}$ имеет простые полюсы в точках $x_{1}^{0}$ и $x_{2}^{0}$, возникающие при разрешении нодальной точки кривой $\mathcal{C}$. Плоская метрика $|Q(x)|$ имеет в $x_{i}^{0}$ конические точки с углом $\pi$.

Введем выделенные локальные координаты $\xi_{i}, i=1,2$, в окрестности $x_{i}^{0}$ так, чтобы дифференциал $Q$ в окрестности точки $x_{i}^{0}$ имел вид

$$
Q\left(\xi_{i}\right)=\frac{\left(d \xi_{i}\right)^{2}}{\xi_{i}} \quad \text { при } \quad x \rightarrow x_{i}^{0} .
$$

Точки $x_{i}^{0}, i=1,2$, являются точками ветвления канонического накрытия $\widehat{\mathcal{C}}$ с локальными параметрами на $\widehat{\mathcal{C}}$, заданными как $\hat{\xi}_{i}=\xi_{i}^{1 / 2}$; при этом $v=2 d \hat{\xi}_{i}$ вблизи $x_{i}^{0}$. Таким образом, абелев дифференциал $v=\sqrt{Q}$ не имеет особенностей и не обращается в нуль на $\widehat{\mathcal{C}}$ в точках $x_{i}^{0}$. Плоские координаты в окрестности точек $x_{i}^{0}$ записываются как

$$
z_{i}(x)=\int_{x_{i}^{0}}^{x} v=2 \xi_{i}^{1 / 2},
$$

в результате $Q(x)=\left(d z_{i}\right)^{2}$ вблизи $x_{i}^{0}$. В обратную сторону, $\xi_{i}(x)=z_{i}^{2} / 4$.

Обозначим длины ребер с концами в $x_{i}^{0}$ через $\ell_{i}$ и предположим, что разрезы, оканчивающиеся в точках $x_{i}^{0}$, проходят вдоль этих ребер. 
4.1. Разделение двух одновалентных вершин путем плоской перестройки. Чтобы деформировать риманову поверхность $\mathcal{C}$ в риманову поверхность $\mathcal{C}^{\alpha, \beta}$, которая отвечает дифференциалу ДШ со всеми простыми нулями, зафикисируем некоторый радиус $R$, считая, что $R<\ell_{i}, i=1,2$. Обозначим плоский диск $\left|z_{i}(x)\right|<R$ с центром в $x_{i}^{0}$ через $\mathcal{D}_{R, i}$, введем также обозначение $\mathcal{D}_{R}=\mathcal{D}_{R, 1} \cup \mathcal{D}_{R, 2}$. Вырезав диски $\mathcal{D}_{R, i}, i=1,2$, из $\mathcal{C}$, получим открытую риманову поверхность $\mathcal{C}_{R}$ с двумя дырками периметра $\pi R$ в метрике $|Q|$. Каноническое накрытие $\widehat{\mathcal{C}}$ с вырезанными прообразами дисков $\mathcal{D}_{R, i}$ превращается в двулистное накрытие $\widehat{\mathcal{C}}_{R}$ поверхости $\mathcal{C}_{R}$, которое также является римановой поверхностью с двумя дырками.

Построим деформацию $\mathcal{C}^{\alpha, \beta}$ с двумя вещественными малыми параметрами $\alpha$ и $\beta$ так, чтобы ленточный граф, отвечающий поверхности $\mathcal{C}^{\alpha, \beta}$, имел две трехвалентные вершины (т. е. дифференциал $Q$ имел два простых нуля) вместо двух одновалентных вершин $x_{1}^{0}$ и $x_{2}^{0}$. При такой деформации появляются два новых ребра длины $\alpha$ и $\beta$, в то время как $\ell_{1}$ и $\ell_{2}$ изменяются следующим образом:

$$
\left(\ell_{1}, \ell_{2}\right) \rightarrow\left(\ell_{1}-\frac{1}{2}(\alpha+\beta), \ell_{2}-\frac{1}{2}(\alpha+\beta)\right),
$$

в результате периметры обеих граней, содержащих вершины $x_{1}^{0}$ и $x_{2}^{0}$, остаются неизменными.

Для построения $C^{\alpha, \beta}$ мы присоединим к $\mathcal{C}_{R}$ кольцеобразную область следующего вида. Рассмотрим точку в пространстве $\mathcal{Q}_{0}^{\mathbb{R}}\left([1]^{2},[-3]^{2}\right)$, т. е. квадратичный дифференциал $Q_{0}$ на $\mathbb{C P}^{1}$ с двумя полюсами третьего порядка (которые мы отождествим с $x=0$ и $x=\infty)$, с двумя простыми нулями и вещественными периодами:

$$
Q_{0}(x)=\frac{\left(x-x_{1}\right)\left(x-x_{2}\right)}{x^{3}}(d x)^{2} .
$$

Каноническое накрытие, соответствующее дифференциалу $Q_{0}$, является эллиптической кривой $\widehat{\mathcal{C}}_{0}$ с точками ветвления $x_{1}, x_{2}, 0$ и $\infty$ на плоскости переменной $x$. Мероморфный абелев дифференциал $v_{0}=\sqrt{Q_{0}}$,

$$
v_{0}=\frac{\sqrt{\left(x-x_{1}\right)\left(x-x_{2}\right)}}{x^{3 / 2}} d x,
$$

имеет на этом каноническом накрытии нули второго порядка в $x_{1}$ и $x_{2}$ и полюсы второго порядка в 0 и $\infty$.

Предположим, что разрезы выбираются вдоль горизонтальных геодезических в метрике $\left|Q_{0}\right|$, соединяющих точку 0 с $x_{1}$ и точку $x_{2}$ с $\infty$. Приближаясь к $x_{1}$ вдоль геодезической, исходящей из 0 , мы можем повернуть либо налево (это ребро обозначим через $e_{2}$ ), либо направо (ребро $e_{1}$ ). Длины ребер $e_{1}$ и $e_{2}$ будем обозначать через $\alpha$ и $\beta$ соответственно.

Плоские координаты на $\mathbb{C P}^{1}$ и на $\widehat{\mathcal{C}}_{0}$ задаются как обычные абелевы интегралы от $v_{0}$; начальную точку интегрирования можно выбрать произвольно (кроме 0 и $\infty$ ). Ленточный граф на $\mathbb{C P}^{1}$, отвечающий дифференциалу $Q_{0}$, имеет два ребра конечных длин $\alpha$ и $\beta$, которые двумя разными способами соединяют точки $x_{1}$ и $x_{2}$, и два ребра бесконечной длины, при этом мы нумеруем точки $x_{1}$ и $x_{2}$ так, чтобы эти ребра соединяли 0 с $x_{1}$ и $\infty$ с $x_{2}$ (см. рис. 7 ). 


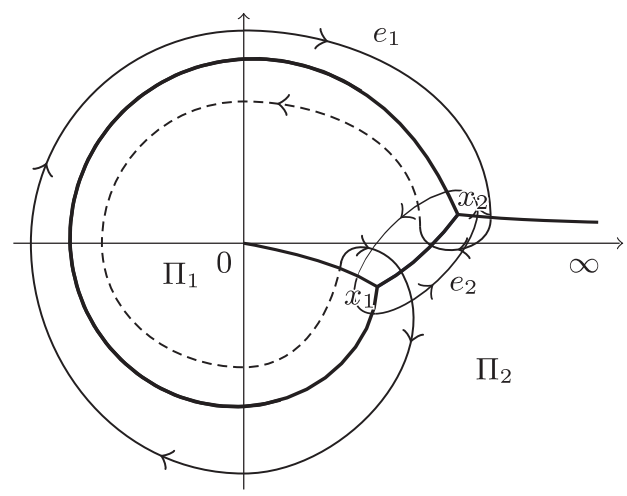

Рис. 7. Разрезы (жирные линии) и другие горизонтальные геодезические на поверхности $\widehat{\mathcal{C}}$. Также показаны ребра $e_{1}$ и $e_{2}$.

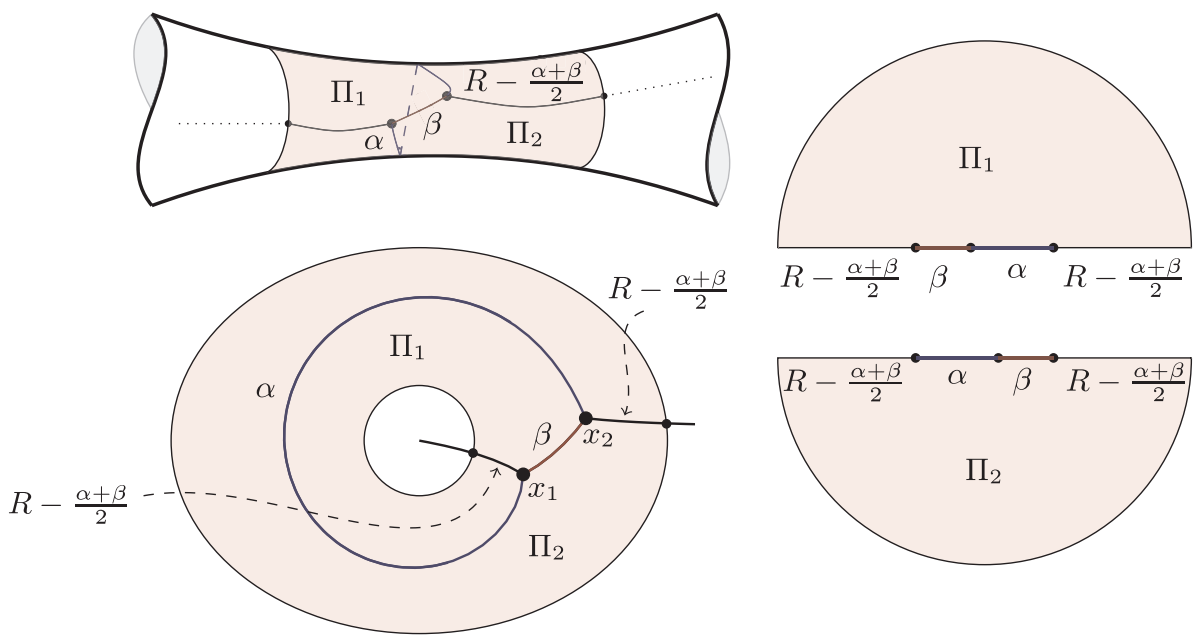

Рис. 8. Закрашенная область на плоскости переменой $x$ конформно отображается в два полудиска в плоских координатах. Траектории на плоскости переменой $x$ построены по результатам численных расчетов.

Рассмотрим кольцевую область $\mathcal{D}_{R}^{\alpha, \beta}$, которая состоит из двух полудисков $\Pi_{1}$ и $\Pi_{2}$ радиуса $R$ в плоской метрике $\left|Q_{0}\right|$, склеенных друг с другом вдоль отрезков длины $\alpha$ и $\beta$ на их диаметрах (см. рис. 8). Оставшиеся части диаметра каждого полудиска (имеющие длины $R-(\alpha+\beta) / 2)$ склеиваются друг с другом на каждом полудиске по отдельности, как показано на рис. 8. Поднятие $\widehat{\mathcal{D}}_{R}^{\alpha, \beta}$ области $\mathcal{D}_{R}^{\alpha, \beta}$ в каноническое накрытие $\widehat{\mathcal{C}}_{0}$ имеет два исходящих разреза, совпадающих с частями диаметров полудисков длины $R-(\alpha+\beta) / 2$. Плоская координата в диске $\Pi_{i}$ задается как $z(x)=\int_{x_{i}}^{x} v$ для $i=1,2$, тогда граница области $\mathcal{D}_{R}^{\alpha, \beta}$ определяется уравнением $|z(x)|=R$. 


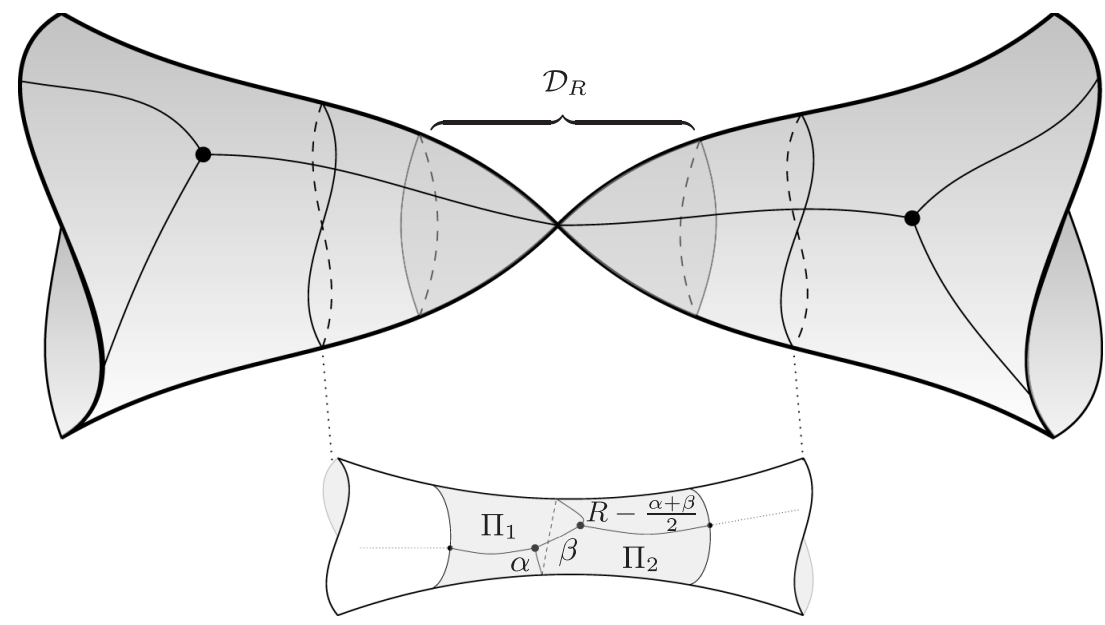

Рис. 9. Разрешение двойной точки $x_{1,2}^{0}$ на поверхности $\mathcal{C}$ путем вклейки кольца с двумя простыми нулями $x_{1}$ и $x_{2}$.

Периметры обеих граничных компонент области $\mathcal{D}_{R}^{\alpha, \beta}$ в плоской метрике $\left|Q_{0}\right|^{2}$ равны $\pi R$. Кроме того, обе граничные компоненты в этой метрике имеют постоянный радиус $R$; разрезы идут вдоль вещественной оси плоской координаты. По аналогии с процедурой разделения пятивалентной вершины мы склеиваем область $\mathcal{D}_{R}^{\alpha, \beta}$ и риманову поверхность $\mathcal{C}_{R}$ с двумя дырками так, чтобы разрезы на $\mathcal{C}_{R}$ склеивались с исходящими разрезами на $\mathcal{D}_{R}^{\alpha, \beta}$.

Таким образом мы получаем гладкую риманову поверхность $\mathcal{C}^{\alpha, \beta}$ рода $g$. Ленточный граф на $\mathcal{C}^{\alpha, \beta}$ получается из ленточного графа для $\mathcal{C}$ путем замены ребер в $\mathcal{C}$, имеющих длины $\ell_{1}$ и $\ell_{2}$, на ребра длины $\ell_{i}-(\alpha+\beta) / 2$ (см. формулу $\left.(4.2)\right)$ и введения двух новых ребер, имеющих длины $\alpha$ и $\beta$. Две одновалентные вершины $x_{1}^{0}$ и $x_{2}^{0}$ на $\mathcal{C}$ при этом заменяются двумя трехвалентными вершинами $x_{1}$ и $x_{2}$ на $\mathcal{C}^{\alpha, \beta}$.

4.2. Конструкция вклейки трубок. Как и в случае разделения точки цикла $W_{5}$, чтобы изучить предел $\alpha, \beta \rightarrow 0$, необходимо рассмотреть вырождение квадратичного дифференциала (4.3) в пределе $x_{i} \rightarrow 0, i=1,2$. Альтернативным образом конструкция вклейки трубок позволяет сохранить поверхности $\mathcal{C}$ и $\mathcal{C}_{0}$ (вместе с соответствующими дифференциалами ДШ) фиксированными, контролируя вырождение с помощью параметра трубки $t$. Появление двух зон вклейки трубки вместо одной - это новая черта, присущая случаю $W_{1,1}$.

Чтобы деформировать поверхность $\mathcal{C}$ в $\mathcal{C}^{\alpha, \beta}$ (и наоборот, изучить предел $\alpha, \beta \rightarrow 0$ для $\left.\mathcal{C}^{\alpha, \beta}\right)$, снова введем параметр трубки $t=\alpha+\beta$ и зададим два параметра

$$
A=\frac{\alpha}{t}=\frac{\alpha}{\alpha+\beta}, \quad B=\frac{\beta}{t}=\frac{\beta}{\alpha+\beta}, \quad A+B=1 .
$$

Как и выше, построим поверхность $\mathcal{C}_{R}$, вырезав из $\mathcal{C}$ два диска радиуса $R=2 t$ (в плоской метрике $|Q|$ ) с центрами в точках $x_{i}^{0}, i=1,2$, при этом выберем $R$ меньше длин $\ell_{i}$ ребер ленточного графа на $\mathcal{C}$, имеющих концами точки $x_{i}^{0}$. Рассмотрим квадратичный дифференциал $Q_{0}$ вида (4.3) такой, что периоды соответствующего 


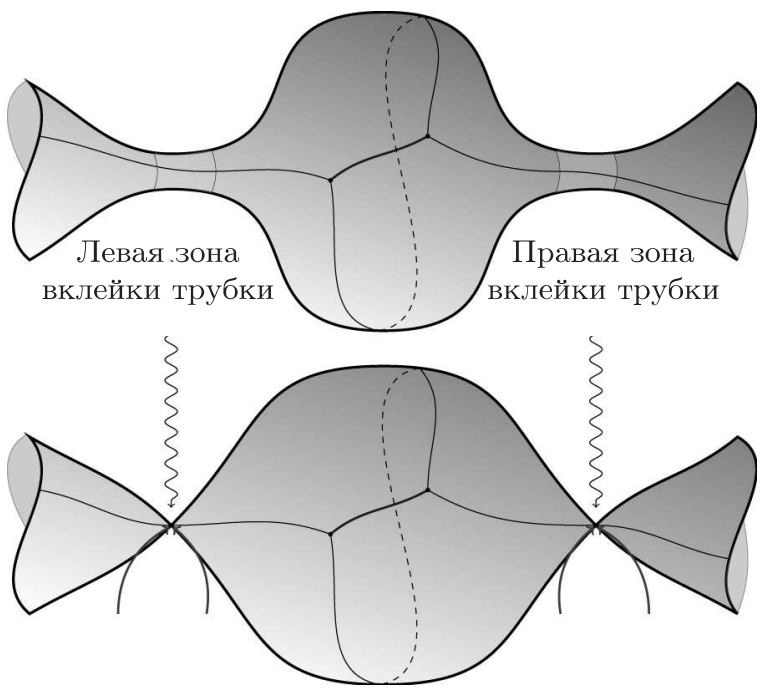

Рис. 10. Разрешение точки границы Концевича $W_{1,1}$ с помощью вклейки трубок: сфера Римана с двумя трехвалентными и двумя одновалентными вершинами вклеивается между двумя одновалентными вершинами с помощью двух трубок.

абелева дифференциала $v_{0}$ по циклам, показанным на рис. 7 , равны $2 A$ и $2 B$ (вместо $2 \alpha$ и $2 \beta$, как выше); при этом в соответствующем ленточном графе на $\mathbb{C P}^{1}$ длины ребер, соединяющих $x_{1}$ и $x_{2}$, равны $A$ и $B$.

Аналогично конструкции предыдущего раздела вырежем из сферы Римана кольцевую область $\mathcal{D}_{2 / t}^{A, B}$, общая длина границ которой в метрике $\left|Q_{0}\right|$ равна $2 \pi / t$. В данном случае диаметр дырок в $\mathcal{C}_{2 t}$ отличается от диаметра границы $\mathcal{D}_{2 / t}^{A, B}$ на множитель $t^{2}$. Вновь обозначим плоские координаты на $\mathcal{C}$ в окрестности $x_{i}^{0}$ через $z_{i}, i=1,2$. Плоские координаты в окрестности границы области $\mathcal{D}_{2 / t}^{A, B}$ обозначим через $w_{i}$; эти координаты нужно отождествить с $t^{2} z_{i}$. Однако выделенными локальными координатами в окрестности $x_{i}$ на $\mathcal{C}$ являются $\zeta_{i}=z_{i}^{2} / 4$. В то же время, на сфере Римана в окрестности границ области $\mathcal{D}_{2 / t}^{A, B}$ мы рассматриваем локальные координаты $\xi_{i}(x)=4\left[\int_{x_{i}}^{x} v_{0}\right]^{-2}$. Координата $z_{i}$ используется как плоская координата в области $\Pi_{i}, i=1,2$.

Теперь рассмотрим на $\mathcal{C}_{2 t}$ два малых кольца $A_{\epsilon}, i=1,2$, заданных неравенствами $2 t<z_{i}(x)<2 t+\epsilon$ (в координатах $\zeta_{i}$ на $A_{\epsilon i}$ ). На сфере Римана рассмотрим кольцо $A_{\epsilon}^{0}$, заданное неравенствами $2 / t<w_{i}(x)<2 / t+\epsilon / t^{2}$ (в координатах $\xi(x)$ на $\left.A_{\epsilon}^{0}\right)$. Тогда отождествление колец $A_{\epsilon i}$ и $A_{\epsilon i}^{0}$, которое требуется в стандартной конструкции вклейки трубки, задается соотношением $\xi_{i}(x) \zeta_{i}(y)=t^{4}$, где $x \in A_{\epsilon i}$, $y \in A_{\epsilon i}^{0}$.

Подход, связанный с конструкцией вклейки двух трубок, проиллюстрирован на рис. 10. Вновь, в отличие от стандартного подхода, параметр трубок $t$ вещественный, а второй параметр деформации $B / A$ спрятан в модуле поверхности $\mathcal{C}_{0}$. 


\section{5. МОДУЛЯРНЫЙ ДИСКРИМИНАНТ $\Delta_{5}$ НА $\mathcal{Q}_{0}^{\mathbb{R}}(-7)$}

\section{1. Пространство $\mathcal{Q}_{0}(-7)$.}

Стратификация. Обозначим через $\mathcal{Q}_{0}(-7)$ комплексное пространство модулей квадратичных дифференциалов $Q$ (здесь и далее мы не пишем индекс 0 у $Q$ ) на $\mathbb{C P}^{1}$, имеющих один полюс порядка 7. Если существует преобразование Мёбиуса $\mu$ такое, что $\mu^{*} Q_{1}=Q_{2}$, то дифференциалы $Q_{1}$ и $Q_{2}$ эквивалентны. Следовательно, без потери общности можно считать, что полюс дифференциала $Q$ находится в точке $x=\infty$; с помощью сдвига и изменения масштаба переменной $x$ дифференциал $Q$ может быть представлен как

$$
Q=\left(x-x_{1}\right)\left(x-x_{2}\right)\left(x-x_{3}\right)(d x)^{2}, \quad x_{1}+x_{2}+x_{3}=0,
$$

где точки берутся с точностью до перестановок. Дифференциалы вида (5.1) эквивалентны друг другу, если и только если множества нулей одного и другого дифференциала суть $\left\{x_{i}\right\}$ и $\left\{\epsilon x_{i}\right\}$, где $\epsilon$ - корень пятой степени из единицы.

Пространство $\mathcal{Q}_{0}(-7)$ имеет комплексную размерность два; оно стратифицируется в соответствии с кратностями нулей квадратичного дифференциала $Q$ следующим образом:

$$
\mathcal{Q}_{0}(-7)=\mathcal{Q}_{0}\left(-7,[1]^{3}\right) \cup \mathcal{Q}_{0}(-7,2,1) \cup \mathcal{Q}_{0}(-7,3) .
$$

Здесь $\mathcal{Q}_{0}\left(-7,[1]^{3}\right)$ - страт комплексной размерности два,

$$
\mathcal{Q}_{0}\left(-7,[1]^{3}\right)=\left\{\left(x_{1}, x_{2}, x_{3}\right) \in \mathbb{C}^{3}: x_{1}+x_{2}+x_{3}=0, x_{i} \neq x_{j}\right\} /\left(S_{3} \times \mathbb{Z}_{5}\right) .
$$

Рассмотрим каноническое накрытие $\widehat{\mathcal{C}}$, определенное дифференциалом $Q$ :

$$
y^{2}=\left(x-x_{1}\right)\left(x-x_{2}\right)\left(x-x_{3}\right) .
$$

Введем на $\widehat{\mathcal{C}}$ некоторый базис канонических циклов $(a, b)$ и определим гомологические координаты путем интегрирования дифференциала $v=y d x$ по этим циклам: $2 A=\int_{a} v, 2 B=\int_{b} v$.

Страт $\mathcal{Q}_{0}(-7,2,1)$ появляется при слиянии двух нулей $x_{i}$. Пусть $x_{2}=x_{3}$, так что $x_{1}=-2 x_{2}$. Тогда точка страта $\mathcal{Q}_{0}(-7,2,1)$ представляется дифференциалом вида

$$
Q=\left(x+2 x_{2}\right)\left(x-x_{2}\right)^{2}(d x)^{2} .
$$

Наконец, страт $\mathcal{Q}_{0}(-7,3)$ содержит только одну точку и представляется квадратичным дифференциалом $Q=x^{3}(d x)^{2}$.

Система координат. Периоды $(A, B)$ можно использовать как локальные координаты в пространстве $\mathcal{Q}_{0}\left(-7,[1]^{3}\right)$ в соответствии со следующей леммой.

Лемма 1. Якобиан перехода от $\left(x_{1}, x_{2}\right) \kappa(A, B)$ имеет вид

$$
\frac{\partial(A, B)}{\partial\left(x_{1}, x_{2}\right)}=2 \pi i \Delta_{5}^{1 / 2}
$$

где $\Delta_{5}$ - модулярный дискриминант,

$$
\Delta_{5}=\left[\left(x_{1}-x_{2}\right)\left(x_{1}-x_{3}\right)\left(x_{2}-x_{3}\right)\right]^{2} .
$$


ДокАЗАТЕЛЬСтво. Имеем

$$
\frac{\partial(A, B)}{\partial\left(x_{1}, x_{2}\right)}=\oint_{a} \frac{\partial v}{\partial x_{1}} \oint_{b} \frac{\partial v}{\partial x_{2}}-\oint_{b} \frac{\partial v}{\partial x_{1}} \oint_{a} \frac{\partial v}{\partial x_{2}} .
$$

Чтобы вычислить этот определитель, мы заменим дифференциал $\partial v / \partial x_{1}$ на

$$
w:=\frac{\partial v}{\partial x_{1}}-\frac{2 x_{1}+x_{2}}{x_{1}+2 x_{2}} \frac{\partial v}{\partial x_{2}}=-\frac{\left(2 x_{1}+x_{2}\right)\left(x_{1}-x_{2}\right)}{2 \sqrt{\left(x-x_{1}\right)\left(x-x_{2}\right)\left(x-x_{3}\right)}} d x
$$

определитель при этом не меняется. Теперь вычисление выражения (5.8) сводится к билинейным соотношениям Римана между голоморфным дифференциалом $w$ и $\partial v / \partial x_{2}$, при этом единственный вклад дается вычетом в $x=\infty$. Его нетрудно найти, получаем (если использовать $t=1 / \sqrt{z}$ как локальный параметр в окрестности бесконечной точки)

$$
\frac{\partial v}{\partial x_{2}}=\left(\frac{x_{1}+2 x_{2}}{t^{2}}+\mathcal{O}(1)\right) d t, \quad w=\left(\left(2 x_{1}+x_{2}\right)\left(x_{1}-x_{2}\right)+\mathcal{O}(t)\right) d t
$$

что приводит к (5.6). Лемма доказана.

Канонический базис $(a, b)$ определяется с точностью до преобразований из группы $S L(2, \mathbb{Z})$. Поэтому периоды $2 A, 2 B$, как и периоды $2 \omega_{1}=\int_{a} v_{0}, 2 \omega_{2}=\int_{b} v_{0}$ голоморфного дифференциала $v_{0}=d x / y$, тоже определяются с точностью до действия группы $S L(2, \mathbb{Z})$.

Группа $S L(2, \mathbb{Z})$ действует на отношение $\kappa=B / A$ так же, как на период $\sigma=\omega_{2} / \omega_{1}$ эллиптической кривой $\mathcal{C}$. Но в отличие от $\sigma$, для которого всегда выполнено условие $\operatorname{Im} \sigma>0$ (в частности, $\omega_{1}$ и $\omega_{2}$ не могут одновременно быть вещественными), для $\kappa$ такого условия не существует.

Сопоставляя период $\sigma$ соответствующего канонического накрытия и дифференциал $Q$, мы получаем естественное расслоение пространства $\mathcal{Q}_{0}\left(-7,[1]^{3}\right)$ над модулярной кривой $\Omega$. Слой можно отождествить с $\mathbb{C}^{*} / \mathbb{Z}_{5}$, поскольку $\mathbb{C}^{*}$-действие $\left(x_{1}, x_{2}, x_{3}\right) \mapsto\left(\lambda x_{1}, \lambda x_{2}, \lambda x_{3}\right), \lambda \in \mathbb{C}^{*}$, оставляет $\sigma$ инвариантным, но при этом, вообще говоря, приводит в новую точку пространства $\mathcal{Q}_{0}\left(-7,[1]^{3}\right)$, если только $\lambda$ не является корнем пятой степени из единицы, $\lambda^{5}=1$.

5.2. Вещественный срез пространства $\mathcal{Q}_{0}^{\mathbb{R}}(-7)$ : кривые Бутру. Эллиптические кривые, соответствующие вещественным периодам дифференциала $v$, известны как кривые Бутру [26], [27].

Пространство $\mathcal{Q}_{0}^{\mathbb{R}}(-7)$ представляет собой вещественный срез пространства $\mathcal{Q}_{0}(-7)$, где все слои содержатся в соответствующих слоях пространства $\mathcal{Q}_{0}(-7)$, и задается условием, что все периоды дифференциала $v=y d x$ на эллиптической кривой $v^{2}=Q$ вещественны. Таким образом, стратификация этого пространства имеет вид

$$
\mathcal{Q}_{0}^{\mathbb{R}}(-7)=\underbrace{\mathcal{Q}_{0}^{\mathbb{R}}\left(-7,[1]^{3}\right)}_{\operatorname{dim}_{\mathbb{R}}=2} \sqcup \underbrace{\mathcal{Q}_{0}^{\mathbb{R}}(-7,2,1)}_{\operatorname{dim}_{\mathbb{R}}=1} \sqcup \underbrace{\mathcal{Q}_{0}^{\mathbb{R}}(-7,3)}_{\operatorname{dim}_{\mathbb{R}}=0} .
$$

Поскольку страт $\mathcal{Q}_{0}(-7,3)$ состоит только из одной точки, $\mathcal{Q}_{0}^{\mathbb{R}}(-7,3)=\mathcal{Q}_{0}(-7,3)$.

Докажем для начала следующую лемму, описывающую конфигурацию точек ветвления кривых Бутру. 
ЛЕмма 2 (讘- и $\mathbb{R}_{+}$-ДЕЙСТВИЕ). Предположим, что все периодъ дифберенииала $v=\sqrt{\left(x-x_{1}\right)\left(x-x_{2}\right)\left(x-x_{3}\right)} d x, x_{1}+x_{2}+x_{3}=0$, вещественны. Тогда все периоды дифференииалов

$$
\sqrt{\left(x-e^{2 i \pi k / 5} x_{1}\right)\left(x-e^{2 i \pi k / 5} x_{2}\right)\left(x-e^{2 i \pi k / 5} x_{3}\right)} d x, \quad k=1, \ldots, 4,
$$

$u$

$$
\sqrt{\left(x-\lambda x_{1}\right)\left(x-\lambda x_{2}\right)\left(x-\lambda x_{3}\right)} d x, \quad \lambda \in \mathbb{R}_{+},
$$

также вещественны.

ДокАзАтЕЛьство. Под действием преобразования $x_{i} \mapsto \lambda e^{2 i \pi k / 5} x_{i}, i=1,2,3$, периоды изменяются как $P \mapsto \lambda^{5 / 2} e^{i \pi k} P$. Следовательно, периоды умножаются на вещественное число и остаются вещественными.

Лемма 3. Имеют место следующие утверждения.

1. Если $Q \in \mathcal{Q}_{0}^{\mathbb{R}}\left(-7,[1]^{3}\right)$, то точки $x_{1}, x_{2}$ и $x_{3}$ не лежат на одной прямой, проходящей через иентр плоскости переменной $x$.

2. Если $Q \in \mathcal{Q}_{0}^{\mathbb{R}}(-7,2,1)$, то $\arg x_{1}, \arg x_{2}$ принимают значения из множества $\{2 i \pi k / 5, k=0, \ldots, 4\}, a \arg x_{3}=\arg x_{i}+\pi, i=1,2$.

ДокАзАТЕльство. 1. Покажем, что условие, согласно которому все $x_{i}$ лежат на одной прямой $e^{i \theta} \mathbb{R}$, несовместно с тем, что все периоды дифференциала $v$ вещественны. Используя $\mathbb{R}_{+}$-масштабирование, без потери общности можно предположить, что $x_{1}=e^{i \theta}, x_{2}=\lambda x_{1}$ и, следовательно, $x_{3}=-(1+\lambda) x_{1}$ при некотором $\lambda>1$. Тогда периоды дифференциала $v$ задаются интегралами

$$
\oint_{\gamma} v=e^{5 i \theta / 2} \oint_{\gamma} \sqrt{(\tilde{x}-1)(\tilde{x}-\lambda)(\tilde{x}+1+\lambda)} d \tilde{x},
$$

где $\gamma$ - произвольный цикл, идущий вокруг точек ветвления $1, \lambda$ и $-1-\lambda$ в плоскости переменной $\tilde{x}$. Интеграл в (5.13) по пути вокруг разреза $[1, \lambda]$ мнимый, а интеграл по пути вокруг отрезка $[-1-\lambda, 1]$ вещественный. Поэтому не существует такого значения $\theta$, что оба этих периода одновременно вещественны, если только некоторые точки ветвления не совпадают. Пусть они совпадают, скажем, $\lambda=1$, тогда вещественность выражения $e^{5 i \theta / 2} \int_{-2}^{1}(\tilde{x}-1) \sqrt{(\tilde{x}+2)} d \tilde{x}$ влечет $5 \theta / 2=0(\bmod \pi)$, что доказывает п. 2 леммы.

ЛЕмма 4. Пусть $Q \in \mathcal{Q}_{0}^{\mathbb{R}}(-7,2,1)$ таков, что его двойной ноль лежит на одном из лучей $e^{2 i \pi k / 5} \mathbb{R}_{+}$. Тогда для любой инфинитезимальной деформации дифференuцала $Q$, сохраняющей условие Бутру, двойной ноль расщепляется на два нуля, лежащих по направлениям, образующим угль $\pm \pi / 4$ с лучом $e^{2 i \pi k / 5} \mathbb{R}_{+} ;$иентральный ноль возникает по направлению $\pm 5 \pi / 4$, где угол отсчитьвается от луча $e^{2 i \pi k / 5} \mathbb{R}_{+}$ в положительную сторону.

ДокАзАтельство. Обозначим через $x_{2}, x_{3}$ два простых нуля, возникающих из двойного нуля, а через $A$ - период дифференциала $v$ вдоль малого цикла, окружающего эти нули. Используя $\mathbb{Z}_{5}$-действие (лемма 2), без потери общности можно предположить, что если $x_{2}=x_{3}$, то $\arg x_{2}=\arg x_{3}=2 \pi / 5$ и $\arg x_{1}=7 \pi / 5$. Чтобы 
найти предел величины $\arg \left(x_{3}-x_{2}\right)$ при $x_{3} \rightarrow x_{2}$, поступим следующим образом. Пусть $x_{3}=x_{2}+\delta$ и $x_{1}=-2 x_{2}-\delta$, где $x_{2}(\delta)=x_{0}+\mathcal{O}(\delta)$ при некотором $x_{0}=\left|x_{0}\right| e^{2 \pi i / 5}$. Тогда с точностью до высших порядков по $\delta$

$$
\begin{aligned}
A & = \pm \int_{x_{2}}^{x_{2}+\delta}\left[\left(x-x_{2}\right)\left(x-x_{2}-\delta\right)\left(x+2 x_{2}+\delta\right)\right]^{1 / 2} d x \sim \\
& \sim \pm\left(3 x_{0}\right)^{1 / 2} \int_{x_{0}}^{x_{0}+\delta}\left[\left(x-x_{0}\right)\left(x-x_{0}-\delta\right)\right]^{1 / 2} d x= \pm\left(3 x_{0}\right)^{1 / 2} \frac{i \pi}{8} \delta^{2} .
\end{aligned}
$$

Поскольку $A \in \mathbb{R}$ и $\arg \left(x_{0}\right)=2 \pi / 5$, отсюда мы имеем (при $\delta \rightarrow 0$ )

$$
2 \arg \delta+\frac{\pi}{2}+\frac{\pi}{5} \rightarrow 0(\bmod \pi)
$$

следовательно, $\arg \delta \rightarrow 2 \pi / 5 \pm \pi / 4$ при $\delta \rightarrow 0$. Это доказывает п. 1 леммы.

Для доказательства второго утверждения необходимо проанализировать критические траектории, выходящие из $x_{2}, x_{3}$, при малых $\delta$. Для этого положим

$$
\zeta=e^{-2 i \pi / 5} \frac{x-\left|x_{0}\right|}{|\delta|}, \quad q(\zeta ;|\delta|)=|\delta|^{-4} Q(x),
$$

так что точки $x_{2}, x_{3}$ сопоставляются двум точкам в плоскости переменной $\zeta$, находящимся на единичном расстоянии от начала координат, а точка $x_{1}$ сопоставляется точке, находящейся на расстоянии $\mathcal{O}\left(\delta^{-1}\right)$ от начала координат (см. далее рис. 14). Тогда непосредственное вычисление дает

$$
q(\zeta ;|\delta|)=(|\delta| \zeta+3 r)\left(\zeta^{2}-e^{2 i \arg \delta-4 i \pi / 5}\right) d \zeta^{2} .
$$

Заметим, что $q$ также является квадратичным дифференциалом с вещественными периодами, и структура его траектории имеет ту же топологию, что и в случае дифференциала $Q$ (с точностью до аффинного преобразования). Луч $e^{2 i \pi / 5} \mathbb{R}_{+}$сопоставляется действительной оси на плоскости переменной $\zeta$. Теперь при $|\delta| \rightarrow 0$ (т. е. при $A \rightarrow 0$ ) мы получаем (равномерно по всем компактным множествам в плоскости переменной $\zeta$ )

$$
\lim _{A \rightarrow 0_{+}} q(\zeta ;|\delta|)=3 r\left(\zeta^{2} \pm i\right) d \zeta^{2} .
$$

Рассмотрим случай знака плюс в правой части, случай знака минус рассматривается аналогично. Простой анализ структуры геодезических в метрике, заданной модулем квадратичного дифференциала (5.16), показывает, что траектория, которая ведет к $\operatorname{Re} \zeta=-\infty$, выходит из $e^{5 i \pi / 4}$ (см. рис. 14 ниже). Таким образом, центральный ноль $x_{2}$ - это тот ноль, который сопоставляется $e^{5 i \pi / 4}$. Лемма доказана.

Вещественность периодов дифференциала $v$ на $\widehat{\mathcal{C}}$ позволяет соединить точки $x_{1}$, $x_{2}$ и $x_{3}$ двумя горизонтальными траекториями дифференциала $v$. Тогда один из нулей (скажем, $x_{2}$ ) соединяется с $x_{1}$ и $x_{3}$. Два других нуля $x_{3}$ и $x_{1}$ нумеруются в соответствии со значениями своих аргументов: двигаясь против часовой стрелки вокруг начала координат, мы полагаем $\arg x_{2}<\arg x_{3}<\arg x_{1}$.

Все периоды дифференциала $v$ вещественны. С учетом замечания 1 можно задать $v$ так, что оба интеграла

$$
A=\int_{x_{2}}^{x_{3}} v, \quad B=\int_{x_{2}}^{x_{1}} v
$$


положительны; здесь контуры интегрирования проходят по горизонтальным траекториям дифференциала $v$ и отвечают $a / 2$ и $b / 2$ в группе гомологий. Следовательно, $A$ и $B$ являются длинами горизонтальных геодезических, соединяющих $x_{2}$ с $x_{3}$ и $x_{1}$ соответственно.

ЗАмЕчАниЕ 2. В выражениях (3.5) дифференциал выбирается так, чтобы было выполнено условие $A+B=1$. Это требование всегда можно удовлетворить, применяя $\mathbb{R}_{+}$-масштабирование согласно лемме 2 .

Плоская координата $z(x)$ на $\widehat{\mathcal{C}}$ задается как интеграл от $v$ с начальной точкой $x_{2}$,

$$
z(x)=\int_{x_{2}}^{x} v,
$$

где разрез проходит по объединению траекторий $\left[x_{2}, \infty\right),\left[x_{1}, x_{2}\right]$ и $\left[x_{2}, x_{3}\right]$.

Следующая теорема является аналогом теоремы Штребеля, позволяющей восстановить риманову поверхность с проколами, зная длины ребер и топологию соответствующего ленточного графа.

Теорема 1. Имеют место следующие утверждения.

1. Упорядоченная пара длин $(A, B)$ (5.17) определяет взаимно однозначное отображсение пространства $\mathcal{Q}_{0}^{\mathbb{R}}\left(-7,[1]^{3}\right)$ в $\mathbb{R}_{+}^{2}$.

2. Диагональ $A=B$ соответствует кривым Бутру с антиголоморфной инволюцией (локус Бутру-Кричевера). $C$ помощъю $\mathbb{Z}_{5}$-действия из леммы 2 централъный ноль $x_{2}$ можно всегда сделать вещественным и положительным, в то время как остальные нули всегда можно считать комплексно сопряженными: $x_{1}=\bar{x}_{3}$.

3. Границы $A=0$ и $B=0$, будучи отождествленными, соответствуют точкам пространства $\mathcal{Q}_{0}^{\mathbb{R}}(-7,2,1)$. Оставшаяся ненулевая длина параметризует пространство $\mathcal{Q}_{0}^{\mathbb{R}}(-7,2,1)$.

Доказательство. 1. Якобиан $\partial(A, B) / \partial(x, y)$ координат $(A, B)$, заданных формулой (5.17), никогда не равен нулю в силу (5.6). Однако этого недостаточно, чтобы доказать, что данная функция двух переменных глобально взаимно однозначна. Чтобы доказать обратимость, нам нужно восстановить тройку $\left(x_{1}, x_{2}, x_{3}\right)$ единственным образом (с точностью до $\mathbb{Z}_{5}$-действия) по известным периодам $A$ и $B(5.17)$. Зная длины $A$ и $B$ критического графа, мы строим полиэдральную поверхность (т. е. поверхность с плоской метрикой и коническими особенностями) путем склеивания пяти полуплоскостей, как показано на рис. 5. Это однозначно определяет конформную структуру, т. е. $J$-инвариант накрытия $\widehat{\mathcal{C}}$. Кроме того, поскольку мы считаем ноль $x_{2}$ центральным и однозначно нумеруем остальные нули $x_{1}$ и $x_{3}$, можно выбрать пару различных канонических $a$ - и $b$-циклов, предполагая, что $a$ окружает критическую траекторию, соединяющую $x_{2}$ и $x_{3}$. Далее зафиксируем направление $a$-цикла так, что $A=\frac{1}{2} \int_{a} v$. Тогда $b$-цикл окружает критическую траекторию, соединяющую $x_{2}$ и $x_{1}$, и имеет надлежащую ориентацию. Это определяет период $\sigma$ кривой $\widehat{\mathcal{C}}$, отвечающей такой выбранной маркировке Торелли. В свою очередь, это позволяет выразить отношение между точками $x_{i}$ а терминах соответствующих тета-констант по формуле Томэ. Это отношение обозначим через $t$ :

$$
\frac{x_{2}-x_{1}}{x_{3}-x_{1}}=t
$$


Вводя новую переменную $\tilde{x}=\left(x-x_{1}\right) /\left(x_{3}-x_{1}\right)$, мы можем переписать первое из равенств (5.17) как

$$
\left(x_{3}-x_{1}\right)^{5 / 2} \int_{t}^{1} \sqrt{\tilde{x}(\tilde{x}-1)(\tilde{x}-t)} d \tilde{x}=A,
$$

откуда вытекает связь между $x_{1}$ и $x_{3}$ :

$$
x_{3}-x_{1}=\left[\frac{A}{\int_{t}^{1} \sqrt{\tilde{x}(\tilde{x}-1)(\tilde{x}-t)} d \tilde{x}}\right]^{2 / 5},
$$

где правая часть известна с точностью до множителя $e^{4 \pi i / 5}$. Это позволяет сделать вывод о том, что система линейных уравнений (5.19), (5.21) и $x_{1}+x_{2}+x_{3}=0$ определяет все $x_{i}$ однозначно с точностью до $\mathbb{Z}_{5}$-действия. Таким образом доказано, что отображение периодов задает изоморфизм между пространством $\widehat{\mathcal{Q}}_{0}^{\mathbb{R}}\left(-7,[1]^{3}\right)$ и первым квадрантом на плоскости $(A, B)$.

2. Пусть $A=B$. Тогда эллиптическая кривая $\mathcal{C}$ допускает антиголоморфную инволюцию $\tau$, действующую по правилу $z \rightarrow \bar{z}$ в терминах плоской координаты $z$. При этом дифференциал $v=d z$ удовлетворяет соотношению $\overline{v\left(x^{\tau}\right)}=v(x)$. Инволюция $\tau$ должна действовать на плоскости переменной $x$ как отражение относительно прямой, проходящей через начало координат (поскольку $x_{1}+x_{2}+x_{3}=0$, кроме того, точка $x=\infty$ должна оставаться неподвижной при действии $\tau$ ). Таким образом, $\tau$ должна переставлять $x_{1}$ и $x_{3}$, оставляя $x_{2}$ неподвижным (все $x_{i}$ не могут быть инвариантными относительно этой инволюции, так как они не лежат на одной прямой в соответствии с леммой 3, п. 1).

Следовательно, существует вещественное число $\theta$ такое, что $x_{i}=e^{i \theta} x_{i}$, в то время как $\overline{x_{1}^{\prime}}=x_{3}^{\prime}$ и $x_{2}^{\prime} \in \mathbb{R}$. Делая замену переменных $x=e^{i \theta} x^{\prime}$, мы видим, что $\tau$ действует на $x^{\prime}$ по правилу $x^{\prime \tau}=\bar{x}^{\prime}$, следовательно, $\tau(x)=e^{-2 i \theta} \bar{x}$. Тогда

$$
Q(x)=\left(x-e^{i \theta} x_{1}^{\prime}\right)\left(x-e^{i \theta} \bar{x}_{1}^{\prime}\right)\left(x-e^{i \theta} x_{2}^{\prime}\right)(d x)^{2}
$$

и $\overline{Q\left(x^{\tau}\right)}=e^{10 i \theta} Q(x)$. Поскольку мы предположили, что не только дифференциал $Q$, но и $v=d z=\sqrt{Q}$ удовлетворяют уравнению $\overline{v\left(x^{\tau}\right)}=v(x)$, значение $5 \theta$ должно быть кратным $2 \pi$. Кроме того, в силу предположения, что для невырожденных кривых $0 \leqslant \arg x_{2}<2 \pi / 5$, мы заключаем, что $x_{2} \in \mathbb{R}_{+}$и $x_{3}=\bar{x}_{1}$.

3. Рассмотрим точку пространства $\mathcal{Q}_{0}^{\mathbb{R}}(-7,2,1)$, где, например, $x_{2}=x_{3}=r e^{2 i \pi / 5}$ и $x_{1}=-2 r e^{2 i \pi / 5}$. Тогда можно найти в явном виде оставшийся ненулевой период $B$ :

$$
B=\left|\int_{x_{2}}^{x_{1}}\left(x-r e^{2 i \pi / 5}\right) \sqrt{x-2 r e^{-3 i \pi / 5}} d x\right|=\left|\int_{-2 r}^{r}(r-z) \sqrt{z+2 r} d z\right|=\frac{12 \sqrt{3}}{5} r^{5 / 2} .
$$

Это показывает, что период $B \in \mathbb{R}_{+}$является глобальной координатой пространства $\mathcal{Q}_{0}^{\mathbb{R}}(-7,2,1)$. Теорема доказана.

$J$-инвариант кривых Бутру лежит на кривой $\mathcal{R}_{1}$, результат численного расчета которой показан на рис. $11 б$. Точка пересечения $\mathcal{R}_{1}$ с вещественной осью переменной $J$ отвечает единственной кривой, которая одновременно является вещественной (т. е. допускает антиголоморфную инволюцию) и кривой Бутру. Для этой кривой 


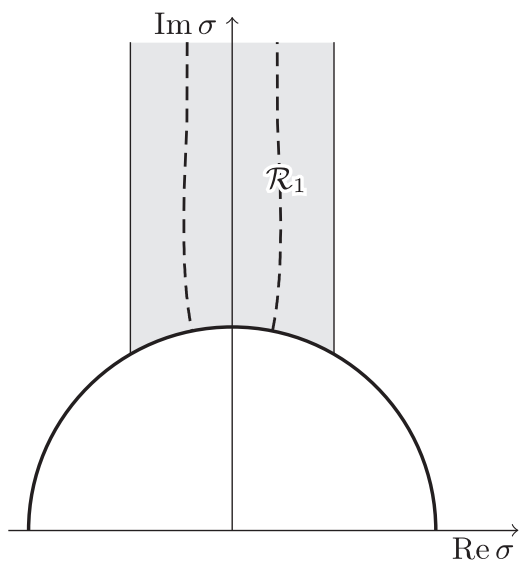

a

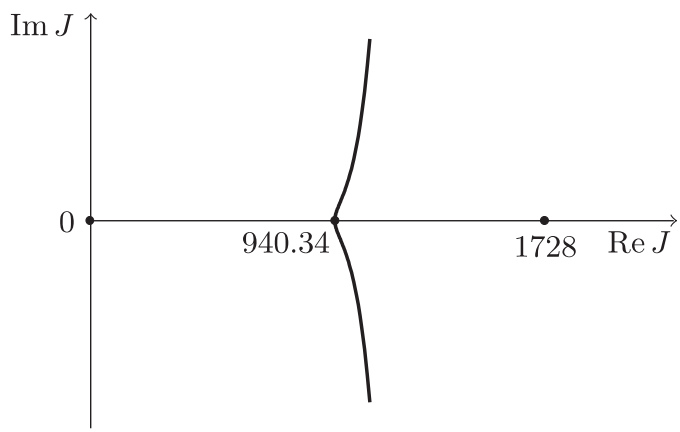

б

Рис. 11. Пространство кривых Бутру $\mathcal{Q}_{0}^{\mathbb{R}}(-7)$ расслоено со слоем $\mathbb{R}_{+}$над штриховой кривой $\mathcal{R}_{1}$ в пространстве модулей эллиптических кривых (а). Множество $\mathcal{R}_{1}$ на плоскости $J$-инвариианта (б); точка $J=1728$ соответствует значению $\sigma=i$, а точка $J=0$ - значению $\sigma=e^{2 \pi i / 3}$.

(известной как кривая Бутру-Кричевера [23]) значение $J$-инварианта приблизительно равно 940.34. Значения $x_{i}$, отвечающие этой кривой, таковы:

$$
x_{1} \simeq-1-6.492 i, \quad x_{2}=2, \quad x_{3} \simeq-1+6.492 i
$$

(или любые кратные этим величинами с произвольным положительным множителем). Множество $\mathcal{R}_{1}$ в плоскости периодов $\sigma$ кривой $\mathcal{C}$ показано на рис. $11 \mathrm{a}$.

Обсудим, какие конфигурации нулей $\left(x_{1}, x_{2}, x_{3}\right)$ отвечают заданной паре периодов $(A, B)$. Следующее предложение является обратным к некоторым утверждениям леммы 3 и теоремы 1.

ПреДЛОЖЕНИЕ 1. Предположим, что все периодъ дифберенциала

$$
v=\sqrt{\left(x-x_{1}\right)\left(x-x_{2}\right)\left(x-x_{3}\right)} d x, \quad x_{1}+x_{2}+x_{3}=0,
$$

вещественны. Тогда один из корней $x_{i}$ принадлежит одному из лучей $e^{2 i \pi k / 5} \mathbb{R}_{+}$, если и только если выполнено одно из двух условий:

1) либо $x_{i}$ - двойной корень (т.е. кривая является вырожденной);

2) либо кривая является невырожденной и допускает антиголоморфную инволючию, менящую местами два других корня, т.е. $\widehat{\mathcal{C}}-$ кривая Бутру-Кричевера из n. 2 теоремы 1; в этом случае $x_{i}$ - центральный ноль, который мы обозначаем через $x_{2}$.

ДокАЗАТЕЛЬСтво. Предположим, что кривая $\widehat{\mathcal{C}}$ является кривой Бутру-Кричевера. Тогда в соответствии с п. 2 теоремы 1 центральный ноль принадлежит одному из лучей $e^{2 i \pi k / 5} \mathbb{R}_{+}$. Предположим, что кривая вырожденна, и запишем 
дифференциал как $v=\left(x-x_{2}\right) \sqrt{x+2 x_{2}} d x$. Тогда прямой расчет дает

$$
\int_{x_{2}}^{-2 x_{2}} v=\frac{12 \sqrt{3}}{5} x_{2}^{5 / 2},
$$

и правая часть равенства является вещественной, если и только если $x_{2}$ принадлежит одному из лучей.

Используя $\mathbb{Z}_{5}$-действие и $\mathbb{R}_{+}$-масштабирование из леммы 2 , мы можем считать, что интересующий нас корень $x_{j}=2$. Тогда два других корня (так как сумма равна нулю) должны иметь вид $-1 \pm \rho$ при некотором $\rho \in \mathbb{C}$. Перепишем дифференциал каK

$$
v=\sqrt{(x-2)(x+1+\rho)(x+1-\rho)} d x .
$$

Кривая является вырожденной, если $\rho=0$ или $\rho= \pm 3$. Случай $\rho=0$ исключается, так как тогда периоды не являются вещественными. В обоих случаях $\rho= \pm 3$ периоды вещественны.

Пусть теперь кривая невырожденна, покажем, что $\rho \in i \mathbb{R}$, откуда также будет следовать, что $x_{j}=x_{2}=2$ есть центральный корень.

Чтобы узнать, для каких значений $\rho \neq 0, \rho \neq \pm 3$ оба периода вещественны, рассмотрим интегралы

$$
A_{ \pm}(\rho)=\int_{2}^{-1 \pm \rho} v d x,
$$

такие что $A_{-}(\rho)=A_{+}(-\rho)$. Для доказательства теоремы достаточно показать, что в верхней полуплоскости существует единственное решение системы

$$
\operatorname{Im} A_{+}(\rho)=\operatorname{Im} A_{-}(\rho)=0
$$

и это решение лежит на оси $\operatorname{Re} \rho=0$. При этом функции $A_{+}(\rho)$ и $A_{-}(\rho)$ аналитичны в областях $\mathcal{D}_{+}=\mathbb{C} \backslash(-\infty, 0]$ и $\mathcal{D}_{-}=\mathbb{C} \backslash[0,+\infty)$ соответственно, и обе эти функции удовлетворяют условию симметрии Шварца: $\overline{A_{ \pm}(\bar{\rho})}=A_{ \pm}(\rho)$. Логика дальнейшего доказательства такова.

1. Рассмотрим кривую (возможно, состоящую из нескольких компонент), заданную уравнением $\Phi=0$, где $\Phi=\operatorname{Im} A_{+}(\rho)$; решения системы (5.23) лежат на пересечении двух кривых $\{\Phi=0\}$ и $-\{\Phi=0\}$. С учетом условия симметрии Шварца для функций $A_{ \pm}(\rho)$ достаточно проанализировать верхнюю полуплоскость.

2. Ограничившись верхней полуплоскостью, покажем, что множество $\Phi=0$ содержится в объединении двух секторов $\mathcal{S}_{\mathrm{R}}$ и $\mathcal{S}_{\mathrm{L}}$ :

$$
\{\rho: \Phi(\rho)=0\} \subset \underbrace{\left\{\arg (\rho-3) \in\left[\frac{3 \pi}{5}, \frac{3 \pi}{4}\right]\right\}}_{\mathcal{S}_{\mathrm{L}}} \bigcup \underbrace{\left\{\arg (\rho-3) \in\left[0, \frac{\pi}{4}\right]\right\}}_{\mathcal{S}_{\mathrm{R}}}
$$

(на рис. 12 эти секторы закрашены), и каждый из двух секторов содержит ровно одну гладкую ветвь кривой $\Phi=0$, начинающуюся из точки $\rho=3$.

3. Поскольку

$$
\mathcal{S}_{\mathrm{L}} \cap\left(-\overline{\mathcal{S}_{\mathrm{L}}}\right)=\varnothing=\mathcal{S}_{\mathrm{R}} \cap\left(-\overline{\mathcal{S}_{\mathrm{L}}}\right)=\left(-\overline{\mathcal{S}_{\mathrm{R}}}\right) \cap \mathcal{S}_{\mathrm{L}}
$$




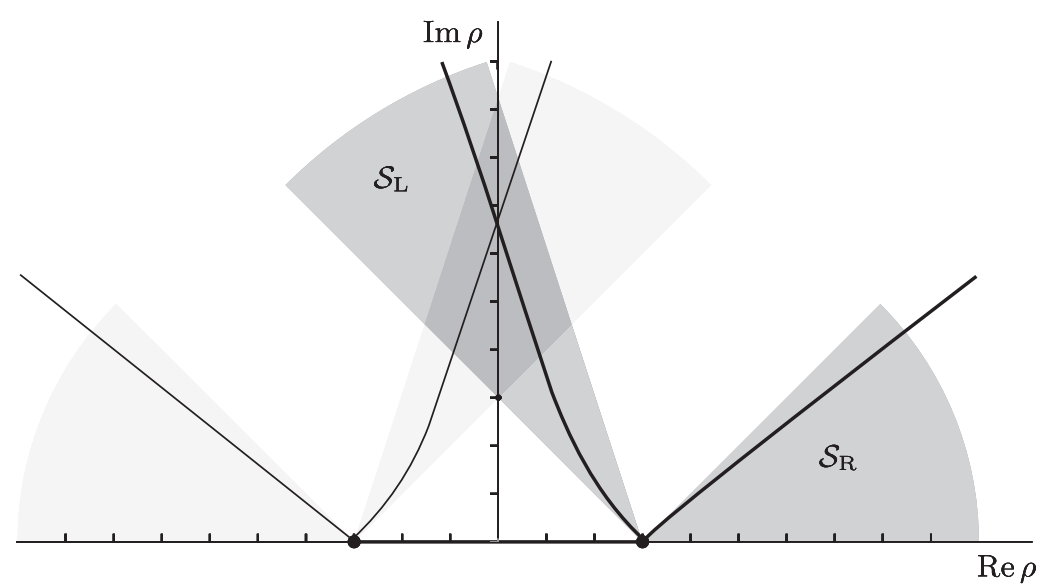

Рис. 12. Множества нулевого уровня функций $\operatorname{Im}\left(A_{+}(\rho)\right)$ (жирные линии) и $\operatorname{Im}\left(A_{-}(\rho)\right)$ (тонкие линии), рассчитанные численно.

все решения уравнений $(5.23)$ должны лежать в области $\mathcal{S}_{\mathrm{L}} \cap\left(-\overline{\mathcal{S}_{\mathrm{L}}}\right)$ (ромбовидной области на рис. 12).

4. Покажем, что существует только одна точка пересечения кривой $\Phi=0$ с положительной мнимой осью и эта точка лежит внутри ромбовидной области.

5. Наконец, докажем, что внутри области $\mathcal{S}_{\mathrm{L}}$ ветвь кривой $\Phi=0$ есть функция от $y=\operatorname{Im} \rho$, для чего покажем, что никакая касательная к слоям $\Phi=$ const не может быть горизонтальной в $\mathcal{S}_{\mathrm{L}}$. Вместе с предыдущим пунктом это сразу влечет единственность решения уравнений (5.23).

Теперь обсудим эти пукты более подробно. Пункт 1 очевиден. Рассмотрим п. 2. Параметризуя интеграл $A_{+}(\rho)$ переменнной $s \in[0,1]$ такой, что $x=2+(\rho-3) \frac{1-s}{1+s}$, получаем для него два выражения:

$$
\begin{aligned}
& A_{+}(\rho)=i(\rho-3)^{2} \int_{0}^{1} \sqrt{\rho+3 s} \frac{\sqrt{s\left(1-s^{2}\right)}}{(1+s)^{4}} d s \\
& A_{+}(\rho)=i(\rho-3)^{5 / 2} \int_{0}^{1} \sqrt{1+3 \frac{s+1}{\rho-3} \frac{\sqrt{s\left(1-s^{2}\right)}}{(1+s)^{4}}} d s .
\end{aligned}
$$

Из представления (5.26) мы видим, что при $|\rho| \rightarrow+\infty$ четыре ветви имеют углы наклона $\pm \pi / 5, \pm 3 \pi / 5$. Кроме того, существует пятая ветвь, состоящая из луча $\rho \in(-\infty,-3]$ (интеграл является чисто мнимым, следовательно, выражение (5.26) вещественное), но эта пятая ветвь не играет никакой роли в наших рассуждениях. Представление (5.26) также полезно при анализе аргумента функции $A_{+}$в конечной области; с этой целью рассмотрим этот аргумент при $\operatorname{Im} \rho>0$. Имеем

$$
\arg \left(A_{+}(\rho)\right)=\frac{\pi}{2}+\frac{5}{2} \arg (\rho-3)+\arg \left(\int_{0}^{1} \sqrt{1+3 \frac{s+1}{\rho-3}} \frac{\sqrt{s\left(1-s^{2}\right)}}{(1+s)^{4}} d s\right) .
$$

Теперь изучим, где аргумент функции $A_{+}$может равняться 0 или $\pi$; если последнее слагаемое в правой части равенства (5.27) отсутствует, то множество $\Phi=0$ будет 
просто состоять из лучей $\arg (\rho-3) \in\{3 \pi / 5, \pi / 5\}$. Учитывая, что интегралы по положительным мерам сохраняют конусы, мы имеем приблизительную оценку, что последнее слагаемое в (5.27) принадлежит интервалу $\left(-\frac{\arg (\rho-3)}{2}, 0\right)$. Из соотношения (5.27) и этой оценки получаем

$$
\frac{\pi}{2}+2 \arg (\rho-3)<\arg A_{+}(\rho)<\frac{\pi}{2}+\frac{5}{2} \arg (\rho-3) .
$$

Чтобы описать секторы $\mathcal{S}_{\mathrm{R}}$ и $\mathcal{S}_{\mathrm{L}}$, нужно проверить, что в дополнение к этим секторам аргумент функции $A_{+}(\rho)$ не может быть равен 0 или $\pi$. Например, в секторе $\arg (\rho-3) \in[\pi / 4,3 \pi / 5]$, лежащем между $\mathcal{S}_{\mathrm{L}}$ и $\mathcal{S}_{\mathrm{R}}$, из неравенства $(5.28)$ мы получаем, что $\pi<\arg A_{+}<2 \pi$ и, следовательно, значения функции $A_{+}$в этом секторе не могут быть вещественными. Аналогично, в секторе $\arg (\rho-3) \in[3 \pi / 4, \pi]$ имеем $2 \pi<\arg A_{+}<3 \pi$, следовательно, функция $A_{+}$и в этом секторе не может принимать вещественные значения.

Итак, кривая $\Phi=0$, т. е. множество, где $\arg A_{+}(\rho) \in\{0, \pi\}$, лежит в объединении секторов $\mathcal{S}_{\mathrm{L}}$ и $\mathcal{S}_{\mathrm{R}}(5.24)$ (см. рис. 12). Кроме того, в каждом из двух секторов существует ровно одна ветвь кривой $\Phi=0$, которая проходит от $\rho=3$ до бесконечности. Это утверждение справедливо в силу того, что множества уровней гармонических функций не могут образовывать ограниченные кривые, если они не окружают особенность; при этом функция $A_{+}(\rho)$ является гармонической во всей верхней полуплоскости. Кроме того, из выражения (5.25) в окрестности $\rho=3$ видно, что существуют четыре ветви кривой $\Phi=0$, исходящие из точки $\rho=3$, углы наклона которых равны $\pm \pi / 4 \pm \pi$. Это доказывает п. 2 нашего плана и, как следствие, п. 3.

Таким образом, все решения системы (5.23) принадлежат ромбовидной области пересечения $\left(-\overline{\mathcal{S}_{\mathrm{L}}}\right) \cap \mathcal{S}_{\mathrm{L}}$ (см. рис. 12$)$, лежащей в полуплоскости $\operatorname{Im} \rho \geqslant 3$.

4. Покажем, что множество $\Phi=0$ при $\operatorname{Im} \rho>3$ пересекается с мнимой ось $i \mathbb{R}_{+}$ переменной $\rho$ в единственной точке $\rho=i \rho_{\star}$. Это означает, что при $\operatorname{Im} \rho<\rho_{\star}$ множество $\Phi=0$ целиком лежит в правой полуплоскости, в то время как ветвь в $\mathcal{S}_{\mathrm{L}}$ при $\operatorname{Im} \rho>\rho_{\star}$ целиком лежит в левой полуплоскости. Другими словами, интересующая нас ветвь не пересекает луч $i \mathbb{R}_{+}$зигзагами несколько раз.

Запишем $A_{+}(i y)$ в более явном виде: мы имеем $\Phi(i y)=\operatorname{Im} A_{+}(i y)$ и

$$
A_{+}(i y)=-i(i y-3)^{2} \int_{0}^{1} \sqrt{3 s+i y} d \mu(s), \quad d \mu(s)=\frac{\sqrt{s\left(1-s^{2}\right)}}{(1+s)^{4}} d s .
$$

Тогда

$$
\Phi(3 i)=\operatorname{Im}\left(-9 i(i-1)^{2} \int_{0}^{1} \sqrt{3 s+3 i} d \mu(s)\right)=-18 \int_{0}^{1} \operatorname{Im} \sqrt{3 s+3 i} d \mu(s)<0
$$

(при этом $\Phi(3 i) \simeq-2.2438)$. Далее, при $y \rightarrow+\infty$ имеем

$$
\Phi(i y) \sim y^{5 / 2} \frac{\sqrt{2}}{2} \int_{0}^{1} d \mu(s) \rightarrow+\infty,
$$

так что при $y \in[3,+\infty)$ существует хотя бы одно решение $\Phi(i y)=0$.

Чтобы показать единственность этого решения, предположим, что существует несколько решений уравнений (5.23), лежащих на мнимой оси; в каждой из этих 
точек оба интеграла $A_{ \pm}$вещественны. Тогда для каждой из этих точек мы имеем $A=B$, и каждая порождает некоторую кривую Бутру-Кричевера. Но из теоремы 1 , п. 2, мы знаем, что кривая Бутру-Кричевера единственна (с точностью до изменения масштаба, которое уже использовалось для фиксации центрального корня в п. 2). Таким образом, приходим к противоречию.

5. Изучим углы наклона векторов, касательных к кривым уровня, и покажем, что касательная не может быть горизонтальной. Другими словами, здесь мы покажем, что в области $\mathcal{S}_{\mathrm{L}}$ аргумент производной $d A_{+} / d \rho$ никогда не равен 0 или $\pi$. Для этого в секторе $S_{\mathrm{L}}=\{\arg (\rho-3) \in[3 \pi / 5,3 \pi / 4]\}$ проанализируем аргумент выражения

$$
\begin{aligned}
\frac{d A_{+}(\rho)}{d \rho}=\oint \frac{d v}{d \rho} d z & =i \rho(\rho-3) \int_{0}^{1} \frac{t d t}{\sqrt{t\left(1-t^{2}\right)\left(\rho+3 \frac{1-t}{t+1}\right)}}= \\
& =-i \rho(\rho-3) \int_{0}^{1} \frac{1}{\sqrt{\rho+3 s}} \frac{(1-s) d s}{(s+1) \sqrt{s\left(1-s^{2}\right)}}
\end{aligned}
$$

Чтобы определить угол наклона кривой $\Phi=0$, рассмотрим $\arg \left(d A_{+} / d \rho\right)$ по модулю $\pi$ (т.е. с точностью до знака). Опять же используем тот факт, что интеграл по положительной мере сохраняет конусы: заметим, что для всех $s \in[0,1], \rho \in S_{\mathrm{L}}$ выполняются следующие неравенства:

$\arg (\rho+3)<\arg (\rho+3 s)<\arg \rho<\arg (\rho-3) \Rightarrow-\arg \rho<-\arg (\rho+3 s)<-\arg (\rho+3)$.

Отсюда

$$
-\frac{\arg \rho}{2}<\arg \left(\int_{0}^{1} \frac{1}{\sqrt{\rho+3 s}} \frac{(1-s) d s}{(s+1) \sqrt{s\left(1-s^{2}\right)}}\right)<-\frac{\arg (\rho+3)}{2} .
$$

Учитывая формулу (5.29) и приведенные выше оценки, находим ограничение снизу:

$$
\arg \frac{d A_{+}}{d \rho}>-\frac{\pi}{2}+\underbrace{\frac{1}{2} \arg \rho}_{\geqslant 0}+\underbrace{\arg (\rho-3)}_{\geqslant 3 \pi / 5} \geqslant \frac{\pi}{10} .
$$

Аналогично можно получить ограничение сверху:

$$
\arg \frac{d A_{+}}{d \rho}<-\frac{\pi}{2}+\underbrace{\arg \rho}_{<\arg (\rho-3)} \underbrace{-\frac{\arg (\rho+3)}{2}}_{<0}+\arg (\rho-3)<-\frac{\pi}{2}+2 \underbrace{\arg (\rho-3)}_{\leqslant 3 \pi / 4} \leqslant \pi .
$$

Конус $\frac{\pi}{10}<\arg \frac{d A_{+}}{d \rho}<\pi$ не содержит вещественной оси, следовательно, касательные наших кривых уровня не могут быть горизонтальными. Таким образом, мы можем параметризовать кривые уровня $\Phi=0$ в секторе $\mathcal{S}_{\mathrm{L}}$ значением мнимой части. Пункт 5 нашего плана и предложение в целом доказаны.

Теперь предположим, что $A \leqslant B$, противоположный случай можно рассмотреть, используя симметрию. Применяя $\mathbb{Z}_{5}$-действие (см. лемму 2 ), сделаем так, что центральный ноль $x_{2}$ лежит в секторе $\mathcal{S}_{2}=\left\{0 \leqslant \arg x_{2}<2 \pi / 5\right\}$. Следующая лемма описывает диапазон аргументов нулей $x_{1}$ и $x_{3}$ при таком предположении. 


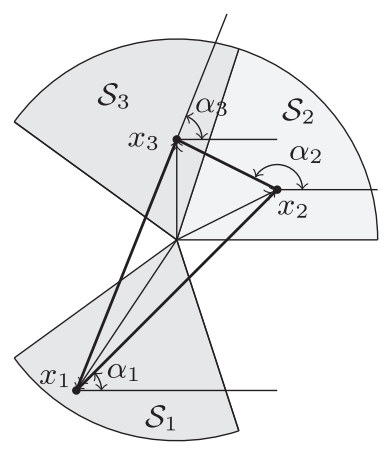

Рис. 13. Секторы $\mathcal{S}_{1}, \mathcal{S}_{2}$ и $\mathcal{S}_{3}$.

ЛЕмма 5. Пусть для иентрального нуля $x_{2}$ выполнено неравенство

$$
0 \leqslant \arg x_{2}<\frac{2 \pi}{5}
$$

а нули $x_{1}$ и $x_{3}$ пронумерованы так, что $A<B$ (т.е. расстояние по метрике $|Q|$ от $x_{2}$ до $x_{1}$ больше расстояния от $x_{2}$ до $\left.x_{3}\right)$. Тогда нули $x_{1}$ и $x_{3}$ лежат в секто$\operatorname{pax} \mathcal{S}_{1} u \mathcal{S}_{3}$ (cм. puc. 13),

$$
\mathcal{S}_{1}=\left\{\frac{6 \pi}{5} \leqslant \arg x_{1} \leqslant \frac{8 \pi}{5}\right\}, \quad \mathcal{S}_{3}=\left\{\frac{2 \pi}{5} \leqslant \arg x_{3} \leqslant \frac{4 \pi}{5}\right\} .
$$

ДокАЗАТЕЛьство использует непрерывность аргумента и предложение 1. Применяя масштабирование с помощью $\mathbb{R}_{+}$-действия, всюду в этой лемме мы считаем без потери общности, что $A+B=1$.

Пусть $A=0, B=1$, тогда из предложения 1 следует, что ноль $x_{3}=x_{2}$ лежит на одном из лучей $e^{2 i \pi k / 5} \mathbb{R}_{+}$. Используя $\mathbb{Z}_{5}$-действие, мы можем положить $k=1$. По мере возрастания величины $A$ корни $x_{2}=x_{3}$ расходятся по направлению, которое найдено в лемме 4 и из которой следует, что эти корни движутся в двух смежных секторах. Рассмотрим случай, когда центральный ноль $x_{2}$ движется в секторе $(0,2 \pi / 5)$; это отвечает знаку минус в правой части равенства (5.16) (другой случай соответствует другой клетке).

Известно, что при малых $A$ корни $x_{i}$ лежат в соответствующих секторах $\mathcal{S}_{i}$, $i=1,2,3$, как на рис. 13. Однако из предложения 1 мы знаем, что никакой из нулей по мере возрастания величины $A$ (напомним, $A+B=1$ ) не может пересечь луч $e^{2 i \pi k / 5} \mathbb{R}_{+}$, если только он не является центральным нулем (что возможно только при $A=B$ ), или если кривая вырождается (когда $A=0$ или $B=0$ ). Таким образом, корни $x_{i}, i=1,2,3$, с необходимостью остаются в указанных секторах. Лемма доказана.

5.3. Вариация величины $\arg \Delta_{5}$ на $\mathcal{Q}_{0}^{\mathbb{R}}(-7)$. Целью данного пункта является изучение вариации аргумента модулярного дискриминанта $\Delta_{5}(5.7)$ на пространстве модулей $\mathcal{Q}_{0}^{\mathbb{R}}(-7)$.

Аргумент дискриминанта $\Delta_{5}$ не изменяется при одновременном умножении всех значений $x_{i}$ на положительную вещественную постоянную. Следовательно, $\arg \Delta_{5}$ 
постоянен вдоль любого луча, исходящего из начала координат в первом квадранте плоскости $(A, B)$. В частности, $\arg \Delta_{5}$ постоянен на каждом из лучей $A=0, B=0$ и $A=B$.

ЛЕмма 6. Пусть, как и выше, аргумент центрального нуля $x_{2}$ лежит между 0

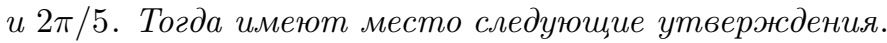

1. Аргументы разностей $x_{i}-x_{j}$ при $A \rightarrow 0$ ведут себя следующим образом:

$$
\begin{aligned}
\arg \left(x_{3}-x_{2}\right) \rightarrow \frac{13 \pi}{20}, \quad & \arg \left(x_{3}-x_{1}\right) \rightarrow \frac{2 \pi}{5}, \quad \arg \left(x_{2}-x_{1}\right) \rightarrow \frac{2 \pi}{5}, \\
& \arg \Delta_{5} \rightarrow \frac{29 \pi}{10} .
\end{aligned}
$$

2. При $A=B$ мы имеем

$$
\begin{array}{cl}
\arg \left(x_{3}-x_{2}\right)=\pi-\alpha, \quad & \arg \left(x_{3}-x_{1}\right)=\frac{\pi}{2}, \quad \arg \left(x_{2}-x_{1}\right)=\alpha, \\
& \arg \Delta_{5}=3 \pi,
\end{array}
$$

где $\alpha$ - угол, который образует прямая, соединяющая точки $x_{2}$ и $x_{3}$ с горизонтальной осъю $(0 \leqslant \alpha \leqslant \pi / 2)$.

3. Аргументы разностей $x_{i}-x_{j}$ при $B \rightarrow 0$ ведут себя следующим образом:

$$
\begin{aligned}
\arg \left(x_{3}-x_{2}\right) & \rightarrow \frac{3 \pi}{5}, \quad \arg \left(x_{3}-x_{1}\right) \rightarrow \frac{3 \pi}{5}, \quad \arg \left(x_{2}-x_{1}\right) \rightarrow \frac{7 \pi}{20}, \\
\arg \Delta_{5} & \rightarrow \frac{31 \pi}{10} .
\end{aligned}
$$

ДокАЗАТЕЛЬСтво. Пределы (5.33) тривиальны: они вытекают из симметрии $x_{1}=\bar{x}_{3}$ при $A=B$ и условия $x_{2} \in \mathbb{R} ;$ это доказывает п. 2 .

Рассмотрим пределы (5.32), возникающие при $A \rightarrow 0$, когда $x_{2} \rightarrow x_{3}$. При $x_{2}=x_{3}$ мы имеем $\arg x_{2}=\arg x_{3}=2 \pi / 5$ и $\arg x_{1}=7 \pi / 5$. Поскольку все $x_{i}$ лежат на одной прямой, проходящей через начало координат, в данном случае $\arg \left(x_{i}-x_{1}\right)=2 \pi / 5$ для $i=2,3$.

Углы между касательными к траекториям точек $x_{2}, x_{3}$, возникающие при разделении двойного нуля, рассчитаны в лемме 4: из нее следует, что при $x_{3} \rightarrow x_{2}$ мы имеем

$$
\lim _{A \rightarrow 0} \arg \left(x_{3}-x_{2}\right)=\frac{2 \pi}{5}+\frac{\pi}{4}=\frac{13 \pi}{20}, \quad \lim _{A \rightarrow 0} \arg \left(x_{3}-x_{1}\right)=\lim _{A \rightarrow 0} \arg \left(x_{2}-x_{1}\right)=\frac{2 \pi}{5},
$$

следовательно,

$$
\arg \Delta_{5}=\lim _{A \rightarrow 0} 2 \arg \left(\left(x_{2}-x_{1}\right)\left(x_{3}-x_{1}\right)\left(x_{3}-x_{2}\right)\right)=\frac{13 \pi}{10}+\frac{8 \pi}{5}=\frac{29 \pi}{10} .
$$

Доказательство соотношений (5.34) аналогично. Лемма доказана.

Теперь мы можем сформулировать первый основной аналитический результат.

Теорема 2. Вариация величинъ $\phi_{5}=\arg \Delta_{5}$ в пространстве $\mathcal{Q}_{0}^{\mathbb{R}}(-7)$ при переходе от границы $B=0 \kappa$ границе $A=0$ равна $\pi / 5$. Другими словами, монодромия величины $\phi_{5}$ при обходе точки $Q=x^{3} d x$ в пространстве $\mathcal{Q}_{0}^{\mathbb{R}}(-7)$ равна $\pi / 5$. 


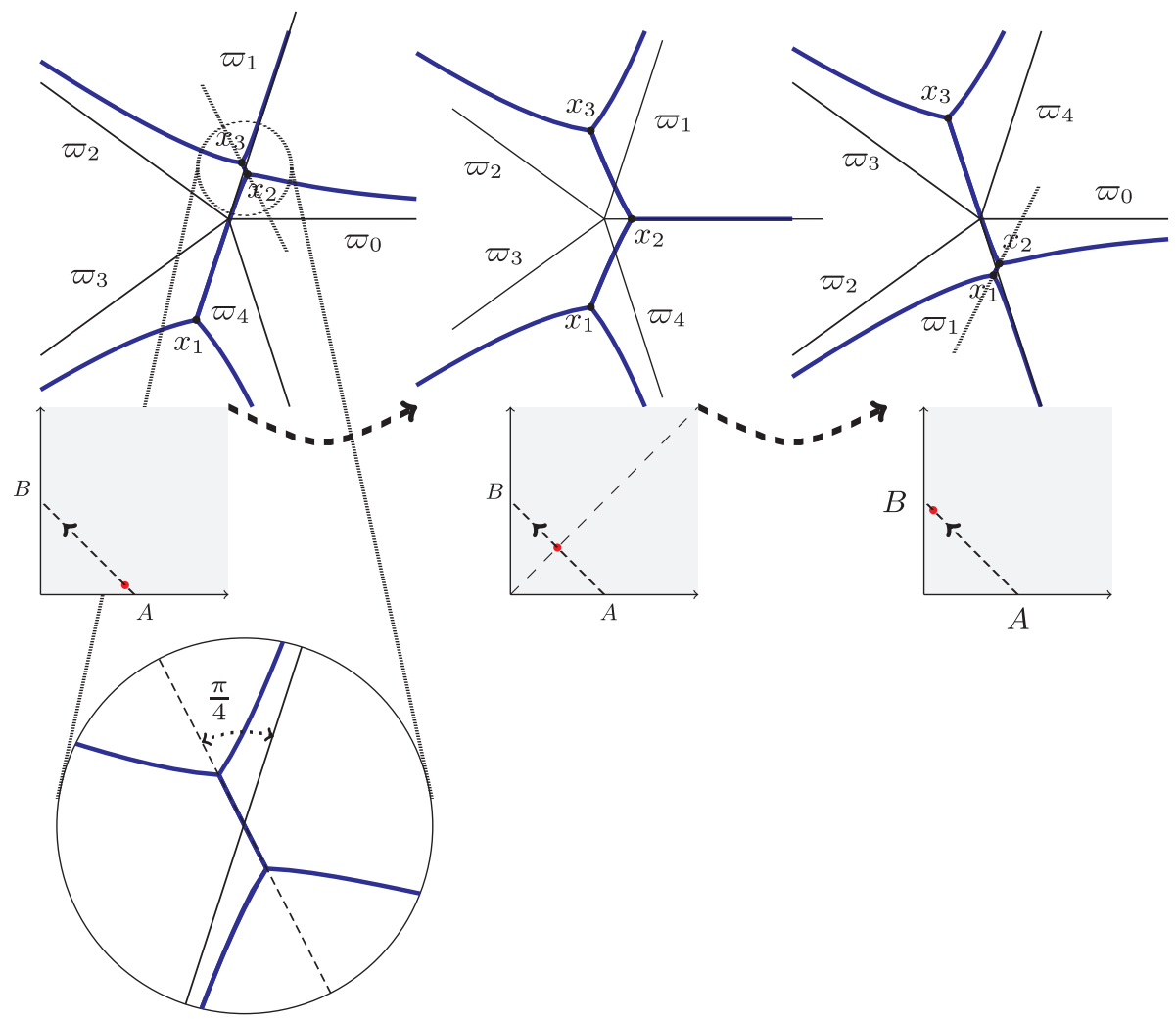

Рис. 14. Движение корней $\left\{x_{i}\right\}$, когда точка $(A, B)$ пересекает клетку, двигаясь из точки $(0,1)$ в точку $(1,0)$ по прямой $A+B=1$. На врезке показан угол, под которым $x_{1}$ и $x_{3}$ приближаются к лучу $\arg x=2 \pi / 5$ при $(A, B) \rightarrow(0,1)$. Лучи $\varpi_{k}$ отвечают $\arg x=2 \pi k / 5, k=0,1,2,3,4$.

ДокАЗАТЕЛЬСТВо теоремы почти немедленно следует из леммы 6. Чтобы показать, что при вычислении вариации не возникает дополнительного вклада, кратного $2 \pi$, достаточно заметить, что с учетом положения нулей $x_{1}$ и $x_{3}$, если $\arg x_{2}$ лежит между 0 и $2 \pi / 5$ (см. рис. (13)), выполнены неравенства

$$
\frac{\pi}{5} \leqslant \arg \left(x_{3}-x_{1}\right) \leqslant \frac{4 \pi}{5}, \quad \frac{2 \pi}{5} \leqslant \arg \left(x_{3}-x_{2}\right) \leqslant \pi, \quad 0 \leqslant \arg \left(x_{2}-x_{1}\right) \leqslant \frac{3 \pi}{5},
$$

которые подтверждают, что вариация величины $\arg \Delta_{5}$ между осью $A=0$ и диагональю $A=B$ в самом деле равна $\pi / 10$. Таким образом, вариация величины $\arg \Delta_{5}$ между осями $A=0$ и $B=0$ равна $\pi / 5$. Теорема доказана.

Заметим, что путь в теореме 2 ориентирован положительно по отношению к форме объема $d A \wedge d B$; выбор этой симплектической формы является естественным в том смысле, что он соответствует индексу пересечения на пространстве $H_{-}$, заданном в (2.1). 


\section{6. АРГУМЕНТЫ ВЕЛИЧИН $\Delta_{1,1}^{ \pm}$В ПРОСТРАНСТВЕ $\mathcal{Q}_{0}^{\mathbb{R}}\left([-3]^{2}\right)$}

В этом разделе мы изучаем пространство $\mathcal{Q}_{0}^{\mathbb{R}}\left([-3]^{2}\right)$, которое представляет собой локальную модель границы Концевича $W_{1,1}$ комбинаторной модели Штребеля пространства $\mathcal{M}_{g, n}$. Мы находим монодромии аргументов величин

$$
\Delta_{1,1}^{+}=x_{1}^{6} x_{2}^{6}\left(x_{1}-x_{2}\right)^{2}, \quad \Delta_{1,1}^{-}=x_{1}^{6} x_{2}^{6}\left(x_{1}-x_{2}\right)^{26},
$$

являющихся естественными аналогами модулярного дискриминанта в $\mathcal{Q}_{0}^{\mathbb{R}}\left([-3]^{2}\right)$. Мотивацией для изучения этих выражений послужила теория тау-функций Бергмана $\tau_{ \pm}$в пространстве квадратичных дифференциалов [21], [18].

6.1. Пространство $\mathcal{Q}_{0}\left([-3]^{2}\right)$. Элементами пространства $\mathcal{Q}_{0}\left([-3]^{2}\right)$ являются квадратичные дифференциалы $Q$ на сфере Римана с двумя полюсами третьего порядка. Комплексная размерность пространства $\mathcal{Q}_{0}\left([-3]^{2}\right)$ равна 2 , и его стратификация имеет следующий вид:

$$
\mathcal{Q}_{0}\left([-3]^{2}\right)=\mathcal{Q}_{0}\left([-3]^{2},[1]^{2}\right) \sqcup \mathcal{Q}_{0}\left([-3]^{2}, 2\right) .
$$

Используя преобразование Мёбиуса, мы можем считать, что полюсы расположены в точках $x=0$ и $x=\infty$; домножение дифференциала $Q$ на константу позволяет представить его как

$$
Q=\frac{\left(x-x_{1}\right)\left(x-x_{2}\right)}{x^{3}}(d x)^{2}, \quad x_{1} \neq x_{2},
$$

где $x_{1}, x_{2}$ - два комплексных параметра.

Таким образом, мы можем провести следующее отождествление пространств:

$$
\mathcal{Q}_{0}\left([-3]^{2},[1]^{2}\right) \simeq\left\{\left(x_{1}, x_{2}\right) \in\left(\mathbb{C}^{*}\right)^{2}: x_{1} \neq x_{2}\right\} / S_{2} .
$$

Введем для каждого $Q \in \mathcal{Q}_{0}\left([-3]^{2},[1]^{2}\right)$ каноническое накрытие $\widehat{\mathcal{C}}$, заданное уравнением $v^{2}=Q$; оно представляет собой эллиптическую кривую $y^{2}=x\left(x-x_{1}\right)\left(x-x_{2}\right)$, снабженную мероморфным дифференциалом

$$
v=\sqrt{\frac{\left(x-x_{1}\right)\left(x-x_{2}\right)}{x^{3}}} d x,
$$

который имеет нули второго порядка на $\widehat{\mathcal{C}}$ в точках $x_{1}, x_{2}$ и полюсы второго порядка в $x=0$ и $x=\infty$.

Выбирая два канонических цикла $(a, b)$ на $\widehat{\mathcal{C}}$ и интегрируя по ним дифференциал $v$, мы получаем периоды $A$ и $B$, которые, как обычно, заданы с точностью до преобразования из группы $S L(2, \mathbb{Z})$.

Следующая лемма показывает, что периоды $(A, B)$ можно использовать как локальные координаты в пространстве $\mathcal{Q}_{0}\left([1]^{2},[-3]^{2}\right)$.

Лемма 7. Якобиан перехода от $\left(x_{1}, x_{2}\right) \kappa(A, B)$ задается формулой

$$
\frac{\partial(A, B)}{\partial\left(x_{1}, x_{2}\right)}= \pm 2 \pi i \frac{x_{1}-x_{2}}{x_{1} x_{2}} .
$$


ДокАЗАТЕЛЬство аналогично доказательству формулы (5.6). А именно, чтобы найти определитель

$$
\frac{\partial(A, B)}{\partial\left(x_{1}, x_{2}\right)}=\oint_{a} \frac{\partial v}{\partial x_{1}} \oint_{b} \frac{\partial v}{\partial x_{2}}-\oint_{b} \frac{\partial v}{\partial x_{1}} \oint_{a} \frac{\partial v}{\partial x_{2}}
$$

где

$$
\frac{\partial v}{\partial x_{1}}=-\frac{1}{2} \frac{1}{x^{3 / 2}}\left(\frac{x-x_{2}}{x-x_{1}}\right)^{1 / 2}, \quad \frac{\partial v}{\partial x_{2}}=-\frac{1}{2} \frac{1}{x^{3 / 2}}\left(\frac{x-x_{1}}{x-x_{2}}\right)^{1 / 2}
$$

заменим $\partial v / \partial x_{1}$ голоморфным дифференциалом

$$
w=\frac{\partial v}{\partial x_{1}}-\frac{b}{a} \frac{\partial v}{\partial x_{2}}=\frac{x_{2}-x_{1}}{2 x_{1}} \frac{d x}{\left[x\left(x-x_{1}\right)\left(x-x_{2}\right)\right]^{1 / 2}}
$$

(это преобразование не меняет определитель). Тогда равенство (6.7) можно получить, применяя билинейные соотношения Римана к голоморфному дифференциалу $w$ и мероморфному дифференциалу $\partial v / \partial x_{2}$, который имеет полюс второго порядка в $x=0$. Найдем вычет функции $\left(\int^{x} w\right) \frac{\partial v}{\partial x_{2}}$ в точке $x=0$ в терминах локального параметра $\sqrt{x}$, отсюда получим $\left(x_{1} x_{2}\right)^{-1}\left(x_{1}-x_{2}\right)$ и в результате придем к (6.6).

6.2. Вещественный срез $\mathcal{Q}_{0}^{\mathbb{R}}\left([-3]^{2}\right)$ : кривые Бутру. Пусть $\mathcal{Q}_{0}^{\mathbb{R}}\left([-3]^{2}\right)$ - пространство кривых Бутру, т. е. срез пространства $\mathcal{Q}_{0}\left([-3]^{2}\right)$, в котором все периоды дифференциала $v=\sqrt{Q}$ являются вещественными. Стратификация пространства $\mathcal{Q}_{0}^{\mathbb{R}}\left([-3]^{2}\right)$ задается как

$$
\mathcal{Q}_{0}^{\mathbb{R}}\left([-3]^{2}\right)=\mathcal{Q}_{0}^{\mathbb{R}}\left([-3]^{2},[1]^{2}\right) \cup \mathcal{Q}_{0}^{\mathbb{R}}\left([-3]^{2}, 2\right),
$$

где страт $\mathcal{Q}_{0}^{\mathbb{R}}\left([1]^{2},[-3]^{2}\right)$ имеет вещественную размерность 2 , а страт $\mathcal{Q}_{0}^{\mathbb{R}}\left(2,[-3]^{2}\right)$ имеет вещественную размерность 1 и отвечает дифференциалам $v=x^{-3 / 2}\left(x-x_{1}\right) d x$, у которых период $\int_{x_{1}^{(1)}}^{x^{(2)}} v=8 x_{1}^{1 / 2}$ веществен (здесь $x_{1}^{(1)}$ и $x_{1}^{(2)}$ - точки, лежащие на различных листах римановой поверхности функции $x^{1 / 2}$, проекции которых на плоскость переменной $x$ совпадают с точкой $\left.x_{1}\right)$, т. е. $x_{1} \in \mathbb{R}_{+}$.

Для определения периодов дифференциала $v$ рассмотрим его горизонтальные траектории, которые имеют вид, показанный на рис. 7: две горизонтальные геодезические всегда соединяют $x_{1}$ и $x_{2}$, в то время как две другие траектории соединяют один из нулей (а именно ноль $x_{1}$ ) с $x=0$ и другой ноль $x_{2}$ с $x=\infty$. Мы обозначаем геодезические следующим образом. Пусть $e_{0}$ - горизонтальная траектория, соединяющая точки 0 и $x_{1}$. Когда мы приближаемся к точке $x_{1}$ вдоль $e_{0}$, мы обозначаем через $e_{1}$ первую, если отсчитывать против часовой стрелки, горизонтальную траекторию, исходящую из точки $x_{1}$, а оставшуюся траекторию обозначаем через $e_{2}$. Обе эти траектории заканчиваются в $x_{2}$. Обозначим длины траекторий $e_{1}$ и $e_{2}$ (в метрике $|Q|$ ) через $A$ и $B$ (см. рис. 7). Аналогично замечанию 2 и лемме 2 нетрудно показать, что всегда можно удовлетворить условие $A+B=1$ из (4.5) с помощью надлежащего $\mathbb{R}_{+}$-масштабирования переменной $x$. 

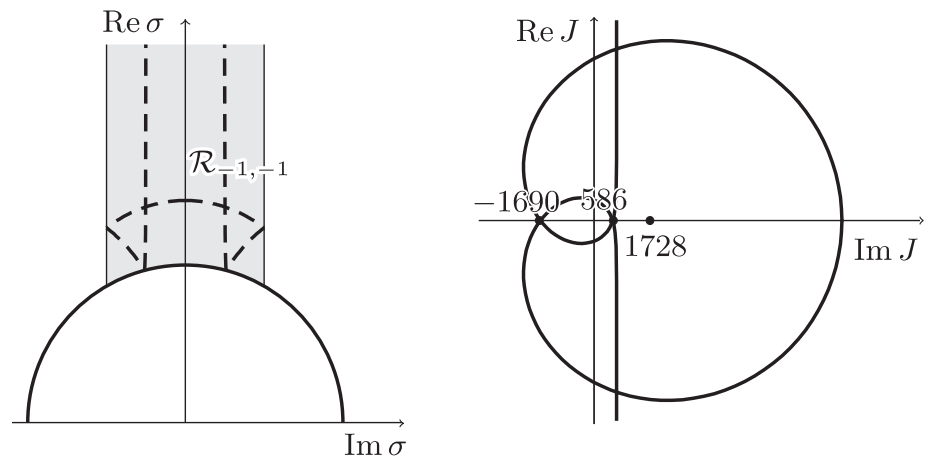

Рис. 15. Множество $\mathcal{R}_{-1,-1}$ на плоскости $J$-инварианта; три вещественные кривые отвечают значениям $J$-инварианта $J_{1} \simeq-1690, J_{2} \simeq 586, J_{3} \simeq 7791$.

Теорема 3. Упорядоченная пара длин $(A, B)$ определяет взаимно однозначное отображение пространства $\mathcal{Q}_{0}^{\mathbb{R}}\left([1]^{2},[-3]^{2}\right)$ в $\mathbb{R}_{+}^{2}$. Полуоси $A=0$ и $B=0$ в $\mathbb{R}_{+}^{2}$ отождествляются с пространством $\mathcal{Q}_{0}^{\mathbb{R}}\left([-3]^{2}, 2\right)$. Оставшийся период определяет взаимно однозначное отображение пространства $\mathcal{Q}_{0}^{\mathbb{R}}\left(2,[-3]^{2}\right)$ в $\mathbb{R}_{+}$. Множество $A=B$ отвечает вещественным кривым, если $x_{1}, x_{2} \in \mathbb{R}$ (численный анализ показывает, что приближенное значение Ј-инварианта для этих кривых равно $J \simeq 7791$ ).

ДокАзАТЕЛьство. Чтобы доказать, что отображение периодов обратимо в пространстве $\widehat{\mathcal{Q}}_{0}^{\mathbb{R}}\left([-3]^{2},[1]^{2}\right)$, необходимо единственным образом восстановить $\left(x_{1}, x_{2}\right)$ по известным периодам $A$ и $B$. Длины $A$ и $B$ задают полиэдральную поверхность (т. е. поверхность с плоской метрикой и коническими особенностями) путем склеивания двух полуплоскостей, как показано на рис. 8. Это однозначно определяет конформную структуру, т. е. $J$-инвариант накрытия $\widehat{\mathcal{C}}$. Кроме того, поскольку фиксирована нумерация нулей $x_{1}$ и $x_{2}$, отношение $t=x_{1} / x_{2}$ задется единственным образом.

Выберем канонический $a$-цикл на $\widehat{\mathcal{C}}$ так, что $2 A=\int_{a} v$. Тогда период

$$
2 A=\int_{\alpha}\left[\frac{\left(x-x_{1}\right)\left(x-x_{2}\right)}{x^{3}}\right]^{1 / 2} d x
$$

после подстановки $\tilde{x}=x / x_{2}$ можно записать как

$$
2 A=x_{2}^{1 / 2} \int_{1}^{t}\left[\frac{(\tilde{x}-t)(\tilde{x}-1)}{\tilde{x}^{3}}\right]^{1 / 2} d \tilde{x},
$$

и это соотношение фиксирует $x_{2}$ единственным образом, поскольку $t$ уже известно.

Таким образом, мы показали, что существует изоморфизм между $\mathcal{Q}_{0}^{\mathbb{R}}\left([1]^{2},[-3]^{2}\right)$ и первым квадрантом $\mathbb{R}_{+}^{2}$ в плоскости $(A, B)$. Теорема доказана.

Периоды $\sigma$ канонических накрытий $\widehat{\mathcal{C}}$, отвечающие точкам из $\mathcal{Q}_{0}^{\mathbb{R}}\left([1]^{2},[-3]^{2}\right)$, принадлежат одномерному подмножеству $\mathcal{R}_{1,1}$ пространства модулей $\mathcal{M}_{1}$, показанному штриховой линией на рис. 15 слева. Множество $\mathcal{R}_{1,1}$ в плоскости $J$-инварианта показано на рис. 15 справа. Оно пересекается с вещественной осью в следующих трех 
точках:

$$
\begin{array}{ll}
J_{1} \simeq-1690, & \left(x_{1} \simeq 1.5538+0.2514 i, x_{2} \simeq 0.1650-0.4893 i\right), \\
J_{2} \simeq 586.3, & \left(x_{1} \simeq 1.2551+0.1883 i, x_{2} \simeq 0.6865-0.2986 i\right), \\
J_{3} \simeq 7791, & \left(x_{1} \simeq 1.8037, x_{2}=-0.3797\right) .
\end{array}
$$

Все эти три точки $J_{i}$ отвечают кривым $\widehat{\mathcal{C}}$ с вещественной инволюцией. Однако только точка $J_{3}$ отвечает кривой Бутру, для которой абелев дифференциал $v=\sqrt{Q}$ инвариантен относительно этой инволюции, т. е. $v(\bar{x})=\overline{v(x)}$.

6.3. Вариации величин $\arg \Delta_{1,1}^{ \pm}$на $\mathcal{Q}_{0}^{\mathbb{R}}\left([-3]^{2}\right)$. Цель данного пункта - изучить вариации аргументов величин (6.1) в пространстве $\mathcal{Q}_{0}^{\mathbb{R}}\left([-3]^{2}\right)$. Формулы (6.1) естественным образом возникают в формализме тау-функций Бергмана [15], [17], [20], [21].

Как и в предыдущем случае, аргументы величин $\Delta_{1,1}^{ \pm}(6.1)$ не изменяются при одновременном умножении всех $x_{i}$ на положительную вещественную постоянную. Следовательно, $\arg \Delta_{1,1}^{ \pm}$постоянны вдоль любого луча, проходящего через начало координат в первом квадранте плоскости $(A, B)$. В частности, $\arg \Delta_{1,1}^{ \pm}$постоянны на каждом из лучей $A=0, B=0$ и $A=B$.

ПрЕДЛОЖЕНИЕ 2. Пусть $Q=\frac{\left(x-x_{1}\right)\left(x-x_{2}\right)}{x^{3}} d x^{2} \in \mathcal{Q}_{0}^{\mathbb{R}}\left([-3]^{2}\right)$, тогда имеют место следующие утверждения.

1. Один из корней $x_{i}$ веществен, если и только если все остальные тоже вещественны. Более того, либо два корня совпадают $\left(x_{1}=x_{2}>0\right)$, либо один из них, (скажем, $\left.x_{2}\right)$ положителен, а другой $\left(x_{1}\right)$ отрицателен, в последнем случае горизонтальные траектории соединяют точку $x_{1}$ с 0 вдоль отрицательной оси и точку $x_{2}$ c $+\infty$ вдоль положительной оси.

2. Если один из корней лежит в верхней полуплоскости, то другой лежит в нижней полуплоскости.

Доказательство этого утверждения чисто техническое, оно содержится в приложении. На рис. 16 показана эволюция точек ветвления $x_{i}$ и горизонтальных траекторий при движении точки $(A, B)$ по прямой от $(1,0)$ до $(0,1)$.

Из предложения 2 вытекает следующее предложение.

ПРЕДЛОЖЕНИЕ 3. Имеют место следующие утверждения.

1. При $A \rightarrow 0$

$$
\arg x_{1} \rightarrow 0, \quad \arg x_{2} \rightarrow 0, \quad \arg \left(x_{2}-x_{1}\right) \rightarrow \frac{\pi}{4} .
$$

2. При $A=B$

$$
\arg x_{1}=0, \quad \arg x_{2}=\pi, \quad \arg \left(x_{2}-x_{1}\right)=0 .
$$

3. $\Pi p u B \rightarrow 0$

$$
\arg x_{1} \rightarrow 2 \pi, \quad \arg x_{2} \rightarrow 0, \quad \arg \left(x_{2}-x_{1}\right) \rightarrow-\frac{\pi}{4} .
$$

ДокАЗАТЕЛЬСТво. 1. При вырождении кривой $\widehat{\mathcal{C}}$ из п. 1 предложения 2 следует, что $\arg x_{i} \rightarrow 0$ и $x_{i} \rightarrow x_{0}>0, i=1,2 ;$ в этом предельном случае плоской координатой 

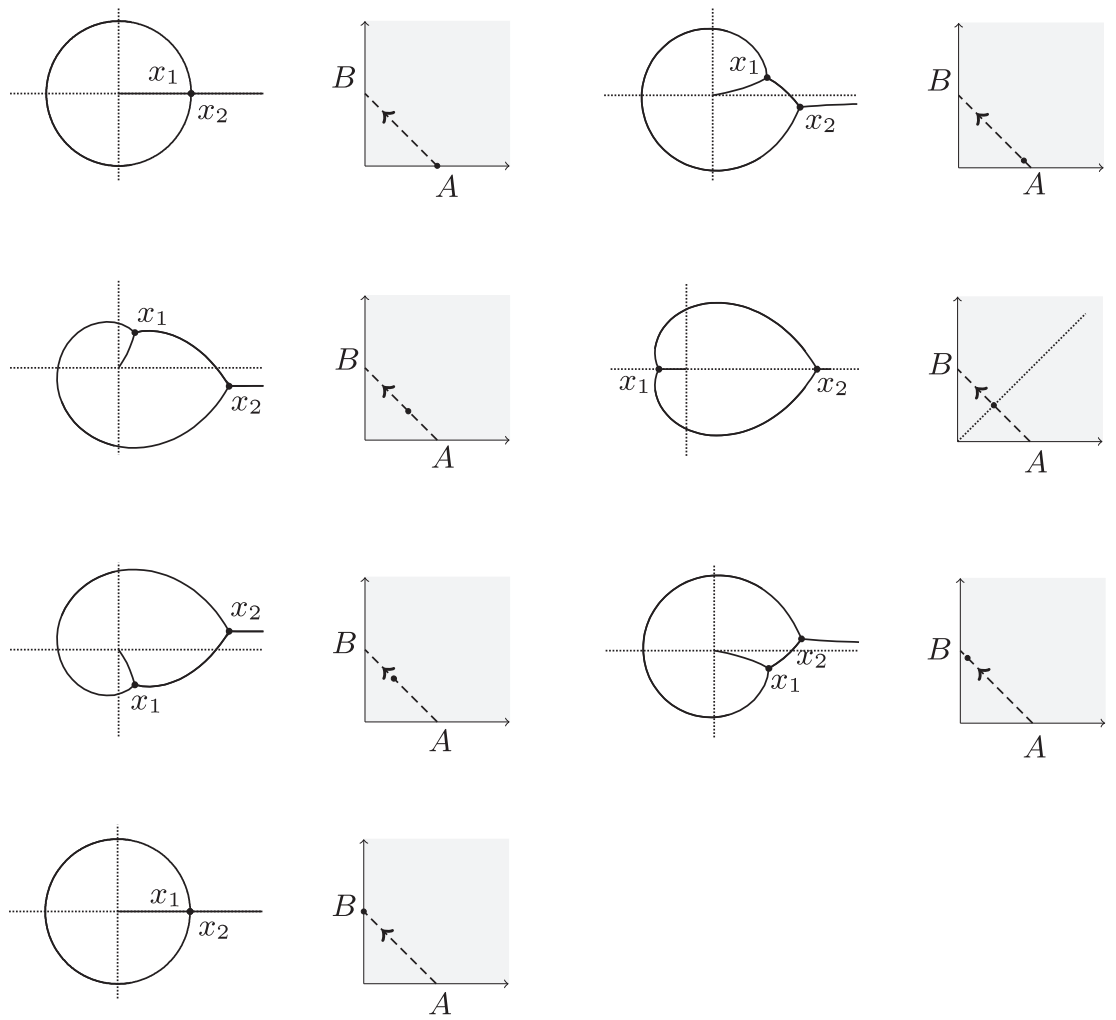

Рис. 16. Когда периоды $(A, B)$ движутся по прямой от точки $(1,0)$ к точке $(0,1)$, ноль $x_{1}$ движется против часовой стрелки вокруг начала координат. Это движение соответствует монодромии при обходе вокруг точки $Q=(d x)^{2} / x$ в пространстве $\widehat{Q}_{0}^{\mathbb{R}}\left([-3]^{2}\right)$.

является $z(x)=\frac{\sqrt{x}}{2}+\frac{x_{0}}{2 \sqrt{x}}$, а критические траектории $\operatorname{Re} z=0$, как легко заметить, имеют вид $\mathbb{R}_{+} \cup\left\{|x|=x_{0}\right\}$.

В силу предложения 2 два корня остаются в противоположных полуплоскостях. При нашем выборе $A, B$ очевидно, что если $A$ убывает, то точка $x_{1}$ смещается в нижней полуплоскости от оси $\mathbb{R}_{-}$, а точка $x_{2}$ смещается в верхней полуплоскости от оси $\mathbb{R}_{+}$. Мы знаем, что при $A \rightarrow 0$ кривая вырождается и оба нуля сходятся к точке на $\mathbb{R}_{+}$. При этом, поскольку их положение ограничено соответствующими полуплоскостями, отсюда немедленно вытекает утверждение о возрастании $\arg x_{i}$, $i=1,2$. Анализ предела $A \rightarrow 0_{+}$полностью идентичен рассуждениям из леммы 4 (которые мы не повторяем), и он показывет, что $\lim _{A \rightarrow 0_{+}} \arg \left(x_{2}-x_{1}\right)=\pi / 4(\mathrm{~cm}$. также численно точный рис. 16).

2. Из п. 2 предложения 2 следует, что при $A=B$ мы имеем $x_{1} \in \mathbb{R}_{-}, x_{2} \in \mathbb{R}_{+}$, следовательно, $\arg \left(x_{2}-x_{1}\right)=0$.

Пункт 3 получается из п. 1 настоящего предложения путем замены $v(x) \mapsto \overline{v(\bar{x})}$.

Наконец, мы готовы сформулировать второй основной аналитический результат. 
Теорема 4. Монодромии величин $\arg \Delta_{1,1}^{+} u \arg \Delta_{1,1}^{-}$в пространстве $\mathcal{Q}_{0}^{\mathbb{R}}\left([-3]^{2}\right)$ при обходе по элементарной несжимаемой петле равны $13 \pi$ и $25 \pi$ соответственно.

ДокАЗАТЕЛЬСтво. Для вычисления вариации $\arg \Delta_{1,1}^{ \pm}$при движении от диагонали $A=B$ к оси $A=0$ достаточно сослаться на предложение 3 . А именно, при $A=B$ мы имеем $\arg x_{1}=-\pi, \arg x_{2}=0, \arg \left(x_{2}-x_{1}\right)=0$, а при $A=0$ мы имеем (в пределе) $\arg x_{1,2}=0, \arg \left(x_{2}-x_{1}\right)=\pi / 4$. Следовательно, вариации равны $\frac{\pi}{2}+6 \pi=\frac{13}{2} \pi$ для $\Delta_{1,1}^{+}$и $\frac{13}{2} \pi+6 \pi=\frac{25}{2} \pi$ для $\Delta_{1,1}^{-}$. Чтобы получить монодромию при пересечении всей клетки, мы должны удвоить эти вариации.

\section{ПРИЛОЖЕНИЕ}

Доказательство предложения 2)Доказательство предложения 2

1. Свойство Бутру (т. е. вещественность всех периодов) инвариантно относительно $\mathbb{R}_{+}$-действия $x \mapsto \lambda x, x_{j} \rightarrow \lambda x_{j}, Q \mapsto \lambda Q, \lambda \in \mathbb{R}_{+}$. Предположим, что один из корней $x_{j}$ является вещественным; в зависимости от его знака, используя вышеуказанное масштабирование, мы можем ограничиться случаями $x_{j}= \pm 1$. Поэтому изучим дифференциалы

$$
v_{+}=\sqrt{\frac{(x-q)(x-1)}{x}} \frac{d x}{x}, \quad v_{-}=\sqrt{\frac{(x-q)(x+1)}{x}} \frac{d x}{x} .
$$

Вырожденный случай. Пусть $q=1$ для $v_{+}$или $q=-1$ для $v_{-}$, тогда интегралы можно вычислить явно:

$$
\int_{1}^{x} v_{+}=2\left(\sqrt{x}+\frac{1}{\sqrt{x}}-2\right), \quad \int_{-1}^{x} v_{-}=2\left(\sqrt{x}-\frac{1}{\sqrt{x}}-2 i\right) .
$$

В первом случае мы легко замечаем, что горизонтальные траектории (где действительная часть интеграла равна нулю) суть $|x|=1$ и $\mathbb{R}_{+}$. Во втором случае отсутствует траектория, соединяющая точку $x=-1$ с самой собой; это видно, если вычислить интеграл по замкнутому пути из $x=-1$ в $x=-1$ вокруг начала координат: результат в самом деле получается мнимый.

Невырожденный случай. Покажем, что условие Бутру заставляет $q$ быть вещественным и иметь противоположный знак по сравнению с $v_{ \pm}$(т. е. $q$ отрицательна в случае $v_{+}$и положительна в случае $\left.v_{-}\right)$. Рассмотрим эти два случая по отдельности.

(. Случай дифференциала $\left.\$ \mathrm{v}_{-}\{+\} \$\right)$ Случай дифференциала $v_{+}$Преобразуем периоды, интегрируя по частям:

$$
\begin{aligned}
H(q) & :=\frac{1}{2} \int_{\gamma_{1}} \sqrt{\frac{(x-1)(x-q)}{x}} \frac{d x}{x} \stackrel{\text { b.p. }}{=} \int_{0}^{1} \frac{q+1-2 x}{\sqrt{x(1-x)(q-x)}} d x, \\
J(q) & :=\frac{1}{2} \int_{\gamma_{q}} \sqrt{\frac{(x-1)(x-q)}{x}} \frac{d x}{x} \underset{x \mapsto q x}{\stackrel{\text { b.p. }}{\leftrightarrows}} \int_{0}^{1} \frac{1+(1-2 x) q}{\sqrt{x(1-x)(1-q x)}} d x,
\end{aligned}
$$




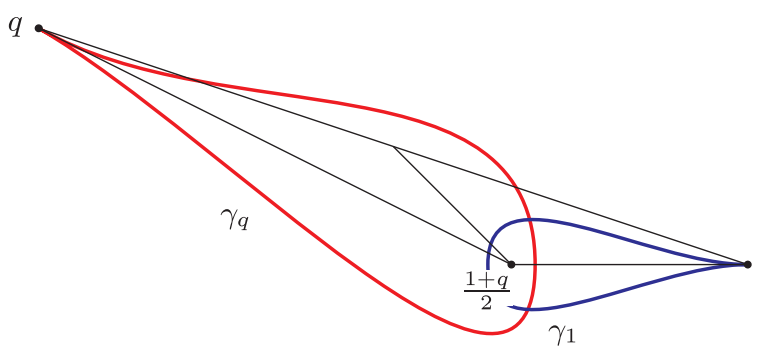

Рис. 17. Контуры интегрирования $\gamma_{1}, \gamma_{q}$.

где контуры интегрирования $\gamma_{1}, \gamma_{q}$ изображены на рис. 17.

Теперь докажем, что уравнения $\operatorname{Im} I(q)=0, \operatorname{Im} J(q)=0$ имеют единственное решение $q^{\star} \in \mathbb{R}_{-}$. Прежде всего покажем, что решение уравнения $\operatorname{Im} J=0$ лежит на луче $q \in(-\infty, 1]$. Функция $J(q)$ заведомо аналитична в области $\mathcal{D}=q \in \mathbb{C} \backslash[1,+\infty)$ и имеет точку ветвления $q=1$. Простой анализ интеграла (П.4) показывает, что:

1) имеют место соотношения

$$
\begin{gathered}
\lim _{q \rightarrow 0+i 0} J(q)=\int_{0}^{1} \frac{d x}{\sqrt{x(1-x)}}=\pi, \quad \lim _{q \rightarrow 1+i 0} J(q)=4, \\
J((-\infty, 1]+i 0)=(-\infty, 4] ;
\end{gathered}
$$

2) при $q>1$ мнимая часть интеграла $J(q)$ (с граничным значением в верхней полуплоскости) положительна (см. оценку (П.7) ниже).

В области $\mathcal{D}$ мы также имеем симметрию Шварца $\overline{J(\bar{q})}=J(q)$, следовательно, достаточно провести анализ только в верхней полуплоскости.

Множество решений уравнения $\operatorname{Im} J=0$ представляет собой объединение, возможно, нескольких гладких дуг, каждая из которых должна допускать бесконечное расширение до тех пор, пока не будет достигнута особенность функции $J(q)$ или ноль производной $J^{\prime}(q)$. Покажем, что $J^{\prime}(q) \neq 0$ в $\mathcal{D}$ с помощью следующих простых выкладок:

$$
J^{\prime}(q)=-\frac{1}{4} \int_{\gamma_{q}} \sqrt{\frac{x-1}{x(x-q)}} \frac{d x}{x} d x \stackrel{\text { b.p. }}{=}-\frac{1}{4 q} \int_{\gamma_{q}} \sqrt{\frac{q-x}{x(1-x)}} d x \underset{z=q t}{\stackrel{\text { b.p. }}{=}}-\frac{1}{2} \int_{0}^{1} \sqrt{\frac{1-t}{t(1-q t)}} d t .
$$

Последний интеграл, очевидно, отличен от нуля при всех $q \in \mathcal{D}$.

Поскольку производная $J^{\prime}(q)$ не имеет нулей в $\mathcal{D}$, всякая ветвь обратного преобразования $J^{-1}(\mathbb{R})$, лежащая в $\mathbb{H}$, должна стремиться к $\infty$ вдоль некоторого направления или быть ограниченной кривой, начинающейся и заканчивающейся в точке $q=1$. Последний случай исключается, потому что $\operatorname{Im} J$ - гармоническая и ограниченная в $q=1$ функция, не имеющая особенностей в $\mathcal{D}$.

Заметим, что если $|q| \rightarrow \infty$ в верхней полуплоскости, то из (П.4) мы получаем, что $J(q) \sim i \sqrt{q} \ln (1-q) \sim \sqrt{-q} \ln (-q)$, а $\operatorname{Re} J(q)$ (в ведущем порядке по $|q|$ ), как нетрудно видеть, не меняет знак при $0<\arg q<\pi$. Следовательно, $J^{-1}(\mathbb{R})$ не имеет ветвей, уходящих на $\infty$. 
Для доказательства указанного выше свойства 2 интеграла (П.4), заметим, что мы имеем для $q>1$

$$
\begin{aligned}
& \operatorname{Im} J(q)=\sqrt{q} \int_{q^{-1}}^{1} \frac{q^{-1}+1-2 x}{\sqrt{x(1-x)\left(x-q^{-1}\right)}} d x= \\
& =\sqrt{q} \int_{q^{-1}}^{\left(q^{-1}+1\right) / 2} \underbrace{\frac{q^{-1}+1-2 x}{\sqrt{x(1-x)\left(x-q^{-1}\right)}}}_{>0} d x+\sqrt{q} \int_{\left(q^{-1}+1\right) / 2}^{1} \underbrace{\frac{q^{-1}+1-2 x}{\sqrt{x(1-x)\left(x-q^{-1}\right)}}}_{<0} d x .
\end{aligned}
$$

Приведем простые оценки двух интегралов, которые показывают, что $\operatorname{Im} J(q)>0$ :

$$
\int_{q^{-1}}^{\left(q^{-1}+1\right) / 2} \frac{q^{-1}+1-2 z}{\sqrt{x(1-x)\left(x-q^{-1}\right)}} d x>\int_{q^{-1}}^{\left(q^{-1}+1\right) / 2} \frac{q^{-1}+1-2 x}{\sqrt{\frac{q^{-1}+1}{2}(1-x)\left(x-q^{-1}\right)}} d x
$$

и

$$
\begin{gathered}
\int_{\left(q^{-1}+1\right) / 2}^{1} \frac{q^{-1}+1-2 x}{\sqrt{x(1-x)\left(x-q^{-1}\right)}} d x>\int_{\left(q^{-1}+1\right) / 2}^{1} \frac{q^{-1}+1-2 x}{\sqrt{\frac{q^{-1}+1}{2}(1-x)\left(x-q^{-1}\right)}} d x \stackrel{x \mapsto 1+\underline{q}^{-1}-x}{\underline{\underline{\underline{y}}}-x} \int_{\left(q^{-1}+1\right) / 2}^{q^{-1}} \frac{q^{-1}+1-2 x}{\sqrt{\frac{q^{-1}+1}{2}(1-x)\left(x-q^{-1}\right)}} d x= \\
=-\int_{q^{-1}}^{(q+1) / 2} \frac{q^{-1}+1-2 x}{\sqrt{\frac{q^{-1}+1}{2}(1-x)\left(x-q^{-1}\right)}} d x .
\end{gathered}
$$

Таким образом, сумма интегралов (П.7) строго положительна. В итоге единственной ветвью решения уравнения $\operatorname{Im} J=0$ является $(-\infty, 1]$. Это завершает доказательство того, что $J(q)$ вещественна только на $(-\infty, 1]$.

Теперь проведем анализ интеграла $H(q)(П .3)$ на вещественной оси переменной $q$ и покажем, что он принимает вещественные значения только при $q \in[1,+\infty) \cup\left\{q^{\star}\right\}$, где $q^{\star}<0$. Для $q \geqslant 1$ подынтегральная функция принимает вещественные значения при $x \in(0,1)$.

Покажем, что $\operatorname{Im} H(q)>0$ для $q \in[0,1)$. В самом деле, при $0<x<q$ подынтегральная функция принимает вещественные значения, и поэтому мнимая часть имеет вид (эти соотношения аналогичны (П.8), (П.9) с заменой $q^{-1} \mapsto q$ )

$$
\begin{aligned}
\operatorname{Im} H(q) & =\int_{q}^{1} \frac{q+1-2 x}{\sqrt{x(1-x)(x-q)}} d x= \\
& =\int_{q}^{(q+1) / 2} \underbrace{\frac{q+1-2 x}{\sqrt{x(1-x)(x-q)}}}_{>0} d x+\int_{(q+1) / 2}^{1} \underbrace{\frac{q+1-2 x}{\sqrt{x(1-x)(x-q)}}}_{<0} d x .
\end{aligned}
$$


Приведем простые оценки двух интегралов, которые показывают, что $\operatorname{Im} H(q)>0$ :

$$
\begin{aligned}
& \int_{q}^{(q+1) / 2} \frac{q+1-2 x}{\sqrt{x(1-x)(x-q)}} d x>\int_{q}^{(q+1) / 2} \frac{q+1-2 x}{\sqrt{\frac{q+1}{2}(1-x)(x-q)}} d x, \\
& \int_{(q+1) / 2}^{1} \frac{q+1-2 x}{\sqrt{x(1-x)(x-q)}} d x>\int_{(q+1) / 2}^{1} \frac{q+1-2 x}{\sqrt{\frac{q+1}{2}(1-x)(x-q)}} d x \stackrel{z \mapsto 1+q-x}{\underline{z}} \\
& z \mapsto \underline{\underline{1+q}}-x-\int_{q}^{(q+1) / 2} \frac{q+1-2 x}{\sqrt{\frac{q+1}{2}(1-x)(x-q)}} d x,
\end{aligned}
$$

таким образом, сумма интегралов (П.10) строго положительна, поэтому $\operatorname{Im} H(q)>0$ при $q \in[0,1)$.

Пусть теперь $q<0$, тогда интеграл

$$
H(q)=i \int_{0}^{1} \frac{x+|q|+(x-1)}{\sqrt{x(1-x)(|q|+x)}} d x
$$

является чисто мнимым. Мнимая часть этой функции монотонна:

$$
\frac{d}{d q} \operatorname{Im} H(q)=-\frac{1}{2} \int_{0}^{1} \frac{|q|+1}{\sqrt{x(1-x)(|q|+x)}(x+|q|)} d x<0 .
$$

Нетрудно видеть, что

$$
\lim _{q \rightarrow-\infty} H(q)=i \int_{0}^{1} \frac{d x}{\sqrt{x(1-x)}}=i \pi,
$$

в то время как при $q \rightarrow 0$ - значения функции $H(q)$ логарифмически расходятся и стремятся к $-\infty$. Следовательно, $H(q)=0$ в единственной точке $q^{\star}<0$. Численный анализ показывает, что $q^{\star} \simeq-0.21048557$.

(. Случай дифференциала $\left.\$ v_{-}\{-\} \$\right)$ Случай дифференциала $v_{-}$Две независимые гомологические координаты задаются интегралами

$$
\begin{aligned}
& \widehat{H}(q):=\frac{1}{2} \int_{\gamma_{-1}} \sqrt{\frac{(x+1)(x-q)}{x}} \frac{d x}{x} \underset{x \mapsto-x}{\stackrel{\text { b.p. }}{=}} \int_{0}^{1} \frac{1-q+2 x}{\sqrt{x(1-x)(q+x)}} d x,
\end{aligned}
$$

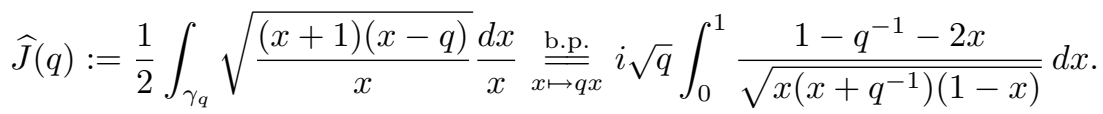

Сначала покажем, что из $\operatorname{Im} q>0$ следует $\operatorname{Im} \widehat{H}(q)<0$, кроме того, $\widehat{H}(q) \in \mathbb{R}$ только при $q>0$. Это нетрудно понять, переписав $\widehat{H}(q)$ как

$$
\widehat{H}(q)=\int_{0}^{1} \frac{1+3 x}{\sqrt{x(1-x)(q+x)}} d x+\int_{0}^{1} \frac{-\sqrt{q+x}}{\sqrt{x(1-x)}} d x .
$$

Предположим, что $\operatorname{Im} q>0$, простой анализ фаз подтверждает, что подынтегральные выражения в обоих интегралах имеют отрицательную мнимую часть. Следовательно, требование $\operatorname{Im} \widehat{H}=0$ влечет $q \in \mathbb{R}$. Предположим, что $q<0$, тогда интегралы в (П.13) (граничное значение берется в $\operatorname{Im} q>0$ ) получают мнимые вклады от 
интегрирования по $x \in[0, \min (1,-q)]$, при этом оба вклада имеют отрицательную мнимую часть. Отсюда заключаем, что $q \in \mathbb{R}_{+}$.

Ограничивая теперь $q$ на $\mathbb{R}_{+}$, мы должны понять, когда $\widehat{J}(q)$ является вещественным для $q \in \mathbb{R}_{+}$. При $q>0$ очевидно, что $\widehat{J}(q) \in i \mathbb{R}$, поэтому мы рассматриваем нули функции $\widehat{J}(q)$ для $q \in \mathbb{R}_{+}$. Элементарный анализ показывает, что функция $\operatorname{Im} \widehat{J}(q)$ возрастает при $q \in \mathbb{R}_{+}$и меняет знак только один раз. Следовательно, значение $\widehat{J}(q)$ вещественно в единственной точке $\tilde{q} r$, которую нужно найти, применяя к предыдущему случаю преобразования гомотетии, так что $\tilde{q}=-1 / q^{\star}>0$.

Наконец, мы покажем, что отрицательный корень связан с началом координат горизонтальной траекторией, поскольку набор всех траекторий должен быть инвариантным относительно преобразования $z \mapsto \bar{z}$. Если траектория, исходящая от одного из корней, проходит вдоль вещественной оси, то она должна быть либо отрезком с одни из концов в нуле, либо лучом, уходящим на бесконечность. Элементарный локальный анализ показывает, что асимптотические направления каждой критической траектории, нисходящей к нулю, идут вдоль $\mathbb{R}_{-}$, а те, которые приближаются к $\infty$, идут вдоль луча $\arg z=0$. Таким образом, с необходимостью отрицательный корень связан с 0 , а положительный $-\mathrm{c}+\infty$.

Выше мы доказали, что либо оба корня имеют ненулевую мнимую часть, либо оба они вещественны. Поэтому, если они не являются вещественными, то они либо находятся в одной (верхней или нижней) полуплоскости, либо в противоположных. Покажем, что имеет место последний случай. Чтобы это увидеть, достаточно вычислить дифференциал по периодам в точке, где оба корня вещественны.

Итак, пусть $v=\sqrt{\left(x-x_{1}\right)\left(x-x_{2}\right) / x} d x$, где $x_{1}=1, x_{2}=q^{\star}$. Мы хотим узнать, что произойдет при бесконечно малой деформации Бутру точек $x_{0}, x_{1}$ (имеется в виду деформация, сохраняющая условие Бутру). Тривиальное вычисление дает (точка обозначает производную по некоторому вещественному параметру деформации $t$ при $t=0)$

$$
\dot{v}=\frac{-x_{1} \dot{x}_{2}-x_{2} \dot{x}_{1}+\left(\dot{x}_{1}+\dot{x}_{2}\right) x}{2 x \sqrt{x\left(x-x_{1}\right)\left(x-x_{2}\right)}} d x,
$$

где $\dot{x}_{1}, \dot{x}_{2}$ ограничены требованием вещественности всех периодов: например, если деформация имеет вид $\dot{A}=1, \dot{B}=0$, тогда потребуем, чтобы периоды функции $\dot{v}$ были заданы, и это однозначно определяет коэффициенты $\dot{x}_{1}, \dot{x}_{2}$. Поскольку мы вычисляем деформацию при $x_{1}=1, x_{2}=q^{\star} \in \mathbb{R}_{-}$, можно записать выражение

$$
\dot{v}=\frac{-\dot{x}_{2}-q^{\star} \dot{x}_{1}+\left(\dot{x}_{1}+\dot{x}_{2}\right) x}{2 x \sqrt{x(x-1)\left(x-q^{\star}\right)}} d x .
$$

Рассмотрим период от $x=1$ до $x=\infty$;

$$
\begin{gathered}
-\left(\dot{x}_{2}+q^{\star} \dot{x}_{1}\right) C_{0}+\left(\dot{x}_{1}+\dot{x}_{2}\right) C_{1}=\alpha \in \mathbb{R}, \\
C_{j}=\int_{1}^{\infty} \frac{x^{j} d x}{\sqrt{x(x-1)\left(x-q^{\star}\right)}}, \quad j=0,1 .
\end{gathered}
$$

Очевидно, что постоянные $C_{j}$ являются вещественными и имеют один знак, зависящий от выбранного знака квадратного корня (скажем, положительны). Кроме того, 
очевидно, что $\left|C_{1}\right|>\left|C_{0}\right|$, поскольку интегрирование ведется по лучу $[1, \infty)$. Взяв мнимую часть выражения (П.16), получаем

$$
-C_{1} \operatorname{Im}\left(q^{\star} \dot{x}_{1}+\dot{x}_{2}\right)+C_{0} \operatorname{Im}\left(\dot{x}_{1}+\dot{x}_{2}\right)=0 \in \mathbb{R} \quad \Rightarrow \quad \operatorname{Im} \dot{x}_{2}=\frac{C_{0}-q^{\star} C_{1}}{C_{0}-C_{1}} \operatorname{Im} \dot{x}_{1} .
$$

Коэффициент $\frac{C_{0}-q^{\star} C_{1}}{C_{0}-C_{1}}$ отрицателен, поскольку $C_{0}, C_{1}$ имеют один знак, величина $q^{\star} \simeq-0.2$ отрицательна и $\left|C_{1}\right|>\left|C_{0}\right|$. При этом начальные направления движения двух корней указывают на противоположные полуплоскости. В силу простой непрерывности точки $x_{1}, x_{2}$ не могут пересекать вещественную ось, не попадая при этом в случай, описанный в предыдущем анализе. Поэтому они остаются в различных полуплоскостях.

Итак, предложение 2 доказано.

Благодарности. Д. Короткин благодарит П. Г. Зографа за интересные обсуждения. Авторы выражают благодарность рецензенту за внимательное чтение рукописи.

\section{Список литературы}

[1] J. Jenkins, "On the existence of certain general extremal metrics", Ann. of Math. (2), 66:3 (1957), 440-453.

[2] K. Strebel, Quadratic Differentials, Ergebnisse der Mathematik und ihrer Grenzgebiete, 5, Springer, Berlin, 1984.

[3] J. Harer, "The cohomology of the moduli space of curves", Theory of Moduli (Montecatini Terme, Italy, June 21-29, 1985), Lecture Notes in Mathematics, 1337, ed. E. Sernesi, Springer, Berlin, 1988, 138-221.

[4] J. L. Harer, "The virtual cohomological dimension of the mapping class group of an orientable surface", Invent. Math., 84:1 (1986), 157-176.

[5] M. Kontsevich, "Intersection theory on the moduli space of curves and the matrix Airy function", Commun. Math. Phys., 147:1 (1992), 1-23.

[6] M. Kontsevich, "Feynman diagrams and low-dimensional topology", First European Congress of Mathematics, v. II: Invited Lectures (Part 2) (Paris, July 6-10, 1992), Progress in Mathematics, 120, eds. A. Joseph, F. Mignot, F. Murat, B. Prum, R. Rentschler, Birkhaüser, Basel, 1994, 97-121.

[7] E. Witten, "Two-dimensional gravity and intersection theory on moduli space", Surveys in Differential Geometry (Harvard University, Cambridge, MA, USA, April 27-29, 1990), Supplement to the Journal of Differential Geometry, 1, eds. C. C. Hsiung, S. T. Yau, H. Lawson, H. Blaine, Jr., AMS, Providence, RI, 1991, 243-310.

[8] K. Igusa, "Graph cohomology and Kontsevich cycles", Topology, 43:6 (2004), 1469-1510.

[9] K. Igusa, "Combinatorial Miller-Morita-Mumford classes and Witten cycles", Algebr. Geom. Topol., 4:1 (2004), 473-520.

[10] G. Mondello, "Riemann surfaces, ribbon graphs and combinatorial classes", Handbook of Teichmüller theory, v. II, IRMA Lectures in Mathematics and Theoretical Physics, 13, ed. A. Papadopoulos, European Math. Soc. Publ. House, Zürich, 2009, 151-215.

[11] G. Mondello, "Combinatorial classes on $\overline{\mathcal{M}}_{g, n}$ are tautological", Int. Math. Res. Notices, 2004:44 (2004), 2329-2390.

[12] R. Penner, "The simplicial compactification of Riemann's moduli space", Topology and Teichmüller spaces (Katinkulta, Finland, 24-28 July, 1995), eds. S. Kojima, Y. Matsumoto, K. Saito, M. Seppälä, World Sci., Singapore, 1996, 237-252. 
[13] E. Arbarello, M. Cornalba, "Combinatorial and algebro-geometric cohomology classes on the moduli spaces of curves", J. Algebraic Geom., 5:4 (1996), 705-749.

[14] D. Zvonkine, Strebel differentials on stable curves and Kontsevich's proof of Witten's conjecture, arXiv: math/0209071.

[15] D. Korotkin, "Solution of matrix Riemann-Hilbert problems with quasi-permutation monodromy matrices", Math. Ann., 329:2 (2004), 335-364.

[16] B. Dubrovin, "Geometry of 2D topological field theories", Integrable systems and quantum groups (Montecatini Terme, June 14-22, 1993), Lecture Notes in Mathematics, 1620, eds. M. Francaviglia, S. Greco, Springer, Berlin, Heidelberg, 1996, 120-348.

[17] A. Kokotov, D. Korotkin, "Tau-functions on spaces of Abelian differentials and higher genus generalization of Ray-Singer formula", J. Differ. Geom., 82:1 (2009), 35-100.

[18] A. Kokotov, D. Korotkin, "Tau-functions on spaces of Abelian and quadratic differentials and determinants of Laplacians in Strebel metrics of finite volume", Preprint No. 46, 2004, arXiv: math/0405042.

[19] A. Kokotov, D. Korotkin, P. Zograf, "Isomonodromic tau function on the space of admissible covers", Adv. Math., 227:1 (2011), 586-600.

[20] D. Korotkin, P. Zograf, "Tau function and moduli of differentials", Math. Res. Lett., 18:3 (2011), 447-458.

[21] D. Korotkin, P. Zograf, "Tau-function and Prym class", Algebraic and Geometric Aspects of Integrable Systems and Random Matrices, Contemporary Mathematics, 593, eds. A. Dzhamay, K. Maruno, V. U. Pierce, AMS, Providence, RI, 2013, 241-261.

[22] M. Bertola, D. Korotkin, "Hodge and Prym tau-functions, Jenkins-Strebel differentials and combinatorial model of $\mathcal{M}_{g, n}$ ", arXiv: 1804.02495.

[23] S.-Y. Lee, R. Teodorescu, P. Wiegman, "Shocks and finite-time singularities in Hele-Shaw flow", Physica D, 238:14 (2009), 1113-1128.

[24] I. M. Krichever, "The $\tau$-function of the universal Whitham hierarchy, matrix models and topological field theories", Commun. Pure Appl. Math., 47:4 (1994), 437-475.

[25] M. Bertola, "Boutroux curves with external field: equilibrium measures without a variational problem", Anal. Math. Phys., 1:2-3 (2011), 167-211.

[26] P. Boutroux, "Recherches sur les transcendantes de M. Painlevé et l'étude asymptotique des équations différentielles du second ordre", Ann. Sci. École Norm. Sup. Ser. 3, 30 (1913), 255-375; "Recherches sur les transcendantes de M. Painlevé et l'étude asymptotique des équations différentielles du second ordre (suite)", 31 (1914), 99-159.

[27] A. S. Fokas, A. R. Its, A. A. Kapaev, V. Yu. Novokshenov, Painlevé Transcendents: The Riemann-Hilbert Approach, Mathematical Surveys and Monographs, 128, AMS, Providence, RI, 2006.

Поступила в редакцию 24.11.2017, после доработки 9.02.2018 